"الأمن الجيوسياسى للقرن الأفريقي و ديناميات القوى الفاعلة:الآفاق المستقبلية لإعادة الصياغة الجيوسياسية ولئية ولئية

"Geopolitical Security for the Horn of Africa and the Dynamics of Active Forces" Future prospects for geopolitical reformulation"

دـ إيهاب عياد-مدرس العلوم السياسية

المستلخص:

هدفت الدراسة إلى الإجابة على الإشكالية الرئيسية والتي مفادها: إلى أي مدى كان ديناميات القوى الفاعلة في منطقة القرن الأفريقي أثرها على الأمن الجيوسياسى للمنطقة؟، من خلال ثلاثة محاور جاء الأول بعنوان: الديناميات الأمنية والجيوسياسية في القرن الأفريقي، ليؤكد فرضية، أن الإقليم الذي يؤثر في القوى العالمية بما ينطوي عليه من خصائص، يكون موضع استقطاب وجذب للعديد من القوى، ليست بالضرورة متواجدة في هذا الإقليم، وجاء المحور الثاني بعنوان: القرن الأفريقي في الحسابات الجيوسياسية الإقليمية والدولية، ليوضح أن تحرك القوى تجاه المنطقة؛ بهدف السيطرة على الموارد، وأن التحركات الخليجية ترتبط بمبررات حماية المصالح الخليجية في المنطقة في ظل تحركات الاستقطاب الخليجية- الإيرانية. ثم تناول المحور الثالث الهندسة الجيوسياسية، والأفاق المستقبلية للقرن الأفريقي ما بعد أزمة كورونا، وتمثلت التوصيات من الناحية البنيوية في: توسيع نطاق المنطقة، وتفعيل دور الاتحاد الأفريقي، وتكوين "هيئة إقليمية لدول القرن الأفريقي" ومن الناحية القيمية: التغلب على نزاعات الهوية، وصياغة مبادرة تتضمن رؤية لنظام "إقليمي أمنى لدول المنطقة". الكلمات المفتاحية: الأمن الجيوسياسى - القرن الأفريقي- ديناميات - الإقليمية - الدولية 


\section{Abstract of the study}

The study aimed to answer the main problem, which is: To what extent did the dynamics of the active forces in the Horn of Africa affect the geopolitical security of the region? Through three axes, the first came under the title: Security and geopolitical dynamics in the Horn of Africa, to confirm the hypothesis, that the region that influences the world powers with the characteristics it implies, is the object of polarization and attraction to many forces, and the second axis came under the title: The Horn of Africa in regional and international geopolitical calculations, to confirm the movement of forces towards the region; With the aim of controlling resources, Then the third axis dealt with geopolitical engineering and future prospects for the Horn of Africa after the Corona crisis, and the recommendation $s$ from the structural point of view were: to expand the scope of the region, activate the role of the African Union, and form a "regional body for the countries of the Horn of Africa" and in terms of value: overcoming identity conflicts and formulating An initiative that includes a vision for a "regional security system for the countries of the region".

Keywords: Geopolitical security- Horn of Africa- Dynamics- RegionalInternational.

يشير المنظور الجيوسياسى لأمن "القرن الأفريقي" - على نحو مبدئي - إلى مقاربة تفاعلاته بما هو أمن إقليمي، يعنى بمجموعة من الدول المتجاورة جغرافياً، إلا أن الواقع يشير إلى غير ذلك؛ حيث امتد المنظور ليشمل نظره أكثر شمولاً وإتساعاً تثير إلى الأثر الذي تتركه السياسات الإقليمية والدولية الأوسع في التأثير والمدى. ولقد كان لتعدد المقاربات التي أعطت أهمية لمنطقة القرن الأفريقي إلى جانب المناطق الأخرى الإقليمية أثرها على تفسير الأهمية الجيوسياسية لهذه المنطقة بصورة أكثر من أي منطقة إقليمه 
أخرى(') ونتيجة لذلك انطلقت المقاربات الجيواستراتيجية لأهمية القرن الأفريقي من منظور مفاده: أن الإقليم الذي تتواجد فيه الدولة يؤثر على حركتها السياسية، وهو ذاته نفس المفهوم الذي انطلقت منه أهداف النظريات الجيوسياسية؛ حيث ترى أن الإقليم الذي يؤثر في القوى العالمية بما ينطوى عليه من خصائص ومزايا تطلعًا نحو السيطرة العالمية، يكون موضع استقطاب، وجذب للعديد من هذه القوى، والتي ليست بالضرورة أن تكون متواجدة في هذا الإقليم(r). كما يشير الواقع الراهن في "القرن الأفريقي" إلى أهمية وخطورة أدوار "الفواعل الإقليمية والدولية" والمقصود بالفواعل هنا الفواعل الدولاتية أي: "الفواعل من الدول" - دون الفواعل غير الدولاتية بصورتيها الرسمية والغير رسمية -؛ حيث تؤكد كافة المؤشرات والمعطيات أن مجمل المتغيرات الراهنة في المنطقة في ظل وجود الفواعل الإقليمية والدولية تتجه نحو تحفيز الصراع واستمراره في المنطقة. ومن ثم فقد أضحت موازين القوة وما يرتبط بها من توازنات دولية وإقليمية، تمثل أحد العوامل المهمة لضرورة إعادة الصياغة الجيوسياسية لأمن المنطقة، وتوسيع رقعة الخارطة الجيوبولتيكية أكثر لتشمل أغلب دول شرق أفريقيا وإقليم البحيرات العظمى، مؤكدة في ذلك على أن تطور الأحداث ومآلاتها المستقبلية في المنطقة، بات يعتمد بشكل أساسي على الفواعل الإقليمية والدولية والتي أصبح دورها واضحاً في الفترة الأخيرة(). ويستخلص مما سبق - أن بواعث الاهتمام بالقرن الأفريقي أتت من منطلق بعدين أولهما: جيوسياسى ويكمن في ما يكتسبه القرن الأفريقي من أهمية حيوية جغرافياً، كون الدول المكون له تطل على المحيط الهندي من ناحية، وتتحكم في المدخل الجنوب للبحر الأحمر ؛يث مضيق باب المندب من ناحية أخرى؛ ومن ثم فإن المنطقة تتحكم في طريق التجارة العالمي، خاصةً تجارة النفط القادمة من دول الخليج، والمتوجهة إلى أوروبا والولايات المتحدة، كما أنها أيضاً تعد كذلك ممراً مهماً لأي تحركات غير اقتصادية قادمة من الغرب في اتجاه منطقة الشرق الأوسط. وتُعزى أهمية هذه المنطقة جغرافياً أيضاً إلى كونها تقع داخل الإقليم الذي أضحى يعرف باسم "قوس الأزمة"، والذي يضم القرن الأفريقي وشبه الجزيرة العربية ومنطقة الخليج. ويتمثل البعد الثاني في: البعد الاقتصادي، والذي يعد ملمحاً آخر لأهمية القرن الأفريقي بالنسبة لبعض القوى الإقليمية والدولية، ومرد ذلك أن دول القرن الأفريقي غنية بالثروات الطبيعية والمعدنية، سواءً النفط أو الذهب أو اليورانيوم أو الغاز الطبيعي. وتمتلك المنطقة ثروة مائية هائلة تجعلها أحد أكبر مخازن المياه في العالم، كما تضم أيضاً كذلك ثروة حيوانية هائلة. 
تأتى أهمية الدراسة منطلق بعدين الأول: جيوسياسى ويكمن في ما يكتسبه القرن الأفريقي من أهمية حيوية جغرافياً، ويتمثل البعد الثاني في: البعد الاقتصادي، والذي يعد ملمحاً آخر لأهمية القرن الأفريقي بالنسبة لبعض القوى الإقليمية والدولية؛ مما جعله موضع استقطاب، وجذب للعديد من هذه القوى، والذي أثر بدوره على الأمن الجيوسياسى للمنطقة. ب أهداف الدراسةة

هدفت الدراسة إلى تحليل الديناميات الأمنية، والجيوسياسية للقرن الأفريقي، وأهميته في الحسابات الجيوسياسية الإقليمية والدولية، وإعادة الصياغة الجيوسياسية للمنطقة بما يحقق الأمن الجيوسياسى الدولي والإقليمي.

\section{ب-الإشكالية الرئيسية للاراسة:}

تمثلت الإشكالية الرئيسية للدراسة في التساؤل الرئيسي لها والذي تمثل في: إلى أي مدى كان لايناميات القوى الفاعلة في منطقة القرن الأفريقي أثرها على الأمن الجيوسياسى للمنطقة ؟، وكيف يمكن إعادة الصياغة الجيوسياسية لهذه المنطقة بما يحقق الأمن الجيوسياسى الإقليمي والدولي؟. ع -حدود الاراسة (المجال الموضوعي - المجال الزمني): يمكن بلورة حدود الدراسة موضوعياً وزمنياً، كما يلي: على مستوى المجال الموضوعي: تتاولت الدراسة موضوع الأمن الجيوسياسى للقرن الأفريقي في ظل ديناميات القوى الفاعلة، وتكمن أهمية الدراسة فيما شهدته هذه المنطقة من العديد من التغيرات الأمنية، والسياسية، والاقتصادية، والاجتماعية، والأزمات التي نتجت عن الديناميات الخارجية فيها؛ حيث تطرق المجال الموضوعي لدراسة وتحليل الديناميات الأمنية والجيوسياسية في المنطقة، والتعرف على القرن الأفريقي في الحسابات الجيوسياسية الإقليمية والدولية، وأهم الملامح المستقبلية للمنطقة في ظل التحديات الجيوسياسية ثم إعادة الصياغة الجيوسياسية بما يتناسب مع ظروف المنطقة. وعلى مستوى المجال الزمني فقد التزمت الدراسة بالفترة الزمنية للأحداث حتى إعداد هذه الدراسة.

ه-الدراسات السابقة:

سعت هذه الدراسة إلى المساهمة بالتحول من التركيز على كون المنظور الجيوسياسى لأمن القرن الأفريقي يعنى بمجموعة من الدول المتجاورة جغرافياً فقط، إلى واقع يشير إلى منظور يشمل نظره أكثر شمولاً واتساعا من خلال التركيز على الأثر الذي تتركه السياسات الإقليمية والدولية الأوسع في التأثير 
والمدى، مسترشدة في ذلك بما رسخت إليه الأدبيات المتزايدة، والتي تصدت لدراسة وتحليل موضوعات الأمن الجيوسياسى أو لأحد ظواهره، وتوضيح مدى تأثرها في ظل بزوغ العديد من الصراعات المتأثرة

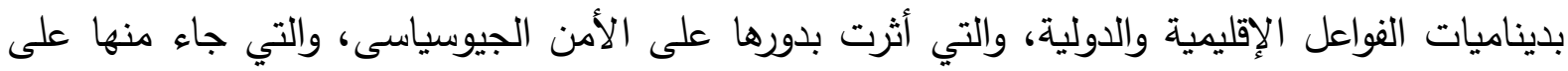

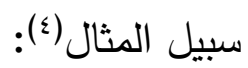

(Amos N. Guiora, 2014; Hans J.Morgenthu,1947; Kornely Kakachia, Stefan Meister \& Benjamin Fricke, 2018; Madonna del Pantano, Lago Patria,(eds).2019; Casier, T. 2016; Sieg, H. M. 2016; Magen, A. 2007; Robert Chase. 1996; D. Helly, G. Galeazzi. 2015).

وكان من أوجه الاختلاف بين الدراسة الحالية والدراسات السابقة: أن الدراسات السابقة ركزت على الصراعات في القرن الأفريقي دون التطرق للأسباب الحقيقية لهذه الصراعات وهى: التواجد والتحرك الإقليمي والدولي في المنطقة، بينما هدفت الدراسة الحالية إلى الحد من ديناميات الفواعل الإقليمية والدولية في المنطقة، لمجابهة مخاطر هذا الانتشار، مع تعظيم الاستفادة منها على المستوى الإقليمي والدولي، وإعادة الصياغة الجيوسياسية لهذه المنطقة، بما يحقق الأمن الجيوسياسى الإقليمي والدولي؛ في ضوء الدراسات السابقة وتحليل العلاقة بين الأمن والجيوسياسية. 7-الإطار المنهجي:

من أجل تحقيق التكامل المنهي، وتوخي المزيد من الدقة، والموضوعية وصولاً للنتائج؛ اتبعت

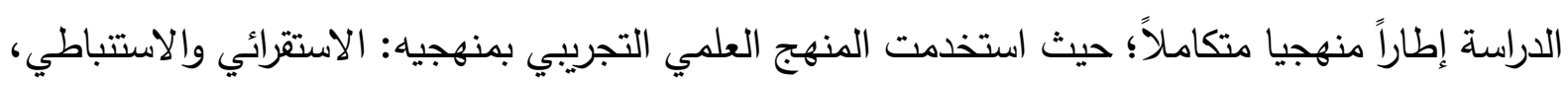
والمنهج التاريخي لمتابعة الظاهرة لفهم الحاضر ، والتتبؤ بالمستقبل مع مراعاة المتغيرات الزمنية، والمكانية والإمكانيات المتاحة في كل فترة. كما أمكن الاستفادة من منهج التحليل النسقى، ومنهج دراسة الحالة(ْ).

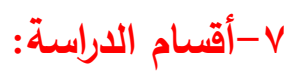
لما كانت هذه الدراسة تسعى للإجابة على تساؤل رئيسي مفاده: "إلى أي مدى كان لديناميات القوى

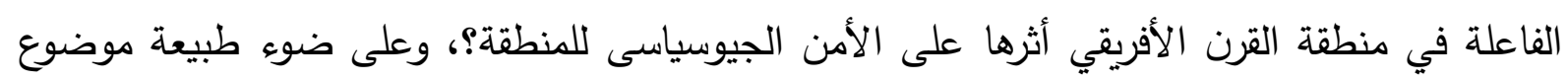
الدراسة والفترة الزمنية الهامة ومقتضياتها، تشمل هذه الدراسة فضلاً عن المقدمة العامة: ثلاثة محاور رئيسية جاء الدحور الأول منها يحمل عنوان: الديناميات الأمنية والجيوسياسية في القرن الأفريقي، وتناول 
المحور الثاني عنوان: القرن الأفريقي في الحسابات الجيوسياسية الإقليمية والدولية على نحو ما هو مبين بالدراسة، ويلحق بهم خاتمة حول نتائج الدراسة، وأهم التوصيات. المحور الأول: الايناميات الأمنية والِيوسياسية في القرن الأقربقي عند النظر للقرن الأفريقي من الناحية الأمنية والجيوسياسية يلاحظ أنه يمثل مركباً صراعياً وأمنياً يتمتع بالخصوصية؛ حيث يجادل البعض في وجود ارتباطات وتأثيرات متبادلة بين الصراعات ومراكز وبؤر التوتر في المنطقة؛ ترجع إلى التتافس على الموارد الطبيعية أو محاولات تحقيق الهيمنة والنفوذ والتي هي في الغالب والأعم سبب تلك الصراعات(؟). كما يرجع البعض الآخر هذه التوترات إلى الخلل الحادث في توازنات القرن الأفريقي والسعي لتتمية المصالح القومية('). ولما كانت الدراسة ترتكز على فرضية مفادها: أن الإقليم الذي يؤثر في القوى الدولية بما ينطوي عليه من خصائص، ومزايا تجعل منهه مطمعاً، وتطلعًا نحو السيطرة العالمية، يكون موضع استقطاب، وجذب للعديد من هذه القوى الإقليمية والدولية، والتي ليست بالضرورة أن تكون متواجدة في هذا الإقليم ـ فقد أرتأت الدراسة أنه من الضروري توضيح مدى أهمية وخطورة أدوار "القوى الإقليمية والدولية"؛ في ضوء ما تؤكده كافة المؤشرات والمعطيات؛ أن مجمل المتغيرات الراهنة في المنطقة في ظل وجود هذه الفواعل تتجه نحو تحفيز الصراع واستمراره في المنطقة. ومن ثم فقد أضحت موازين القوة وما يرتبط بها من توازنات إقليمية ودولية، تمثل أحد العوامل المهمة لضرورة إعادة الصياغة الجيوسياسية لأمن المنطقة، وتوسيع رقعة الخارطة الجيوبولتيكية. ولتوضيح ذلك يتم استعراض الديناميات الأمنية والجيوسياسية في القرن الأفريقي من خلال تناول الموضوعات التالية: أولاً: الأهمية الجيوسيساسية للقرن الأفريقي: لقد تضاربت الدوافع والاعتبارات في إيجاد مفهوم موحد للقرن الأفريقي باعتباره مصطلحا سياسيا يرتبط بشكل وثيق بمصالح الدول الكبرى والتفاعلات الإقليمية والدولية المتسارعة، على اعتبار أن هذه المنطقة تمثل نطاقا جغرافيا، ومنفعاً اقتصادياً وعسكرياً محط تتافس للعديد من الفواعل الدولية والأطماع الخارجية، وذلك من خلال استغلال ما تعانيه المنطقة من تحديات أمنية نتيجة استشراء الاضطرابات وحالة عدم الاستقرار فيها. ويشير المفهوم السياسي للقرن الأفريقي أنه تلك الرقعة الإستراتيجية التي تشمل ثلاثة وحدات سياسية، هي الصومال، جيبوتي وأثيوبيا وتأسس هذا المفهوم انطلاقاً من اعتبارات تاريخية، تجلت في صراعات ممتدة تاريخيا ومتعددة نوعياً سواء كانت إثنية أو قومية أو ثقافية(^)، ويفسر المفهوم 
الجيوبولوتيكى القرن الأفريقي بأنه التخوم الجغرافية التي تعبر عن التحولات المستمرة والمتراكمة في الخارطة الجيوبولوتيكية، فقد كان يعنى بالقرن الأفريقي أنه: المنطقة التي تضم الصومال، وتوسعت رقعتها لتشمل إريتريا، وأثيوبيا، وجيبوتى، والصومال، وتوسعت أكثر لتشمل أغلب دول شرق أفريقيا، وإقليم البحيرات خاصة كينيا والسودان، حتى أصبحت يطلق عليها القرن الأفريقي الكبير أو العظيم (The Greater Horn of Africa) من الاعتبارات السياسية والاقتصادية؛ بحيث يؤكد على أنه طبقاً للتغيرات الحادثة في القرن الأفريقي ونظراً للأهمية الاقتصادية والإستراتيجية التي يتمتع بها، فإنه يشمل المنطقة الشرقية من أفريقيا التي يتوغلها نهر النيل، ومدخل البحر الأحمر الجنوبي وخليج عدن وباب المندب، ومن خلال ذلك تعتبر المنطقة منفذاً بحرياً هاماً باعتبارها أحد الممرات النفطية على المستوى العالمي؛ حيث تعتبر البوابة المركزية لناقلات النفط، والتي تشمل وفقاً لهذا المفهوم عشرة دول من إريتريا شمالاً إلى تنزانيا جنوباً لتضم السودان وجنوب السودان وبورندى وروندا وجيبوتى والصومال وتنزانيا وكينيا وإريتريا(·(). ولقد شكلت منطقة القرن الأفريقي محور اهتمام العديد من القوى الإقليمية والدولية، لاعتبارات جيوبولوتيكية واستراتيجية، راجعة إلى كونها معبر وشريان رئيسي للتجارة الدولية؛ نتيجة امتداد معابرها المائية من باب المندب وصولاً للبحر الأحمر ، كما تعتبر ممراً للتحركات الأمنية لبعض القوى الكبرى المتجهة لمنطقة الثرق الأوسط والخليج العربي، كما حظيت المنطقة باهتمام مجموعة من القوى الدولية كالولايات المتحدة وروسيا والصين لما تمثله من عمق استراتيجي ومنبع ثرواتى ضخم يجعلها أحد أبرز المناطق أهمية على مستوى العالم(') . مما يوضح تمتع المنطقة بميزة تنافسية تؤكد الفرضية التي قامت عليها الدراسة، وهذا ما يؤكده المشهد العام في العقود الأخيرة، وهو مساهمة التحولات الجيواستراتيجية بالعالم في ترقية منطقة القرن الأفريقي كساحة لإعادة ترتيب ميزان القوة بين هذه القوى؛ لتعزيز النفوذ وتأكيد التواجد بالآليات الاقتصادية والعسكرية.

\section{ثانياً: الطبيعة الجيوسياسية وديناميات الصراع في القرن الأفريقي:}

إبان الفترة الاستعمارية تأثر القرن الأفريقي (إريتريا وأثيوبيا وجيبوتى والصومال والسودان وجنوب السودان) بعدد كبير من الحروب الأهلية بين الدول، وأصبحت أوغندا جزء من ديناميات الأمن في المنطقة(ז')، الأمر الذي تشكلت معه خريطة وحدود القرن الأفريقي بصورة متغيرة في حقبة ما بعد الحرب الباردة؛ حيث حدثت تغيرات كبيرة في ملامح القرن الأفريقي؛ أدت إلى إعادة تثكيل أثيوبيا 
والسودان وإنثاء دولتين جديدتين - إريتريا (ع ب مايو ب99 (م)(") ؛ وجنوب السودان (9 يوليو 1 ( ب م) - الأمر الذي تشكل معه حاجة الدول غير الساحلية كأثيوبيا وجنوب السودان للنظر في طرق بحرية بديلة مما شكل منطقة مضطربة ومعرضة لتحديات الصراع(ז'). كما أدت التدخلات الخارجية وظهور ما يسمى بالقوى الصاعدة إلى تفاقم نمط القوة، والسياسة في المنطقة(ء)، واستمرت التحولات في الساحة السياسية للمنطقة في فترة ما بعد الحرب الباردة نتيجة للسياسات، والتدخلات المشتركة في كافة المجالات؛ لتجنب تكرار الحروب في المنطقة، ولتحقيق ما يسمى بالأمن الجيوسياسى وترسيخ مفهوم الدولة القومية الذي رُوج له خلال فترات ما بعد الاستعمار والحرب الباردة؛ إلا أن هذه الأيديولوجية لم تحقق النتائج المتوقعة للاستقرار والسلام في المنطقة(10). والمتمعن في الصراعات الدائرة في القرن الأفريقي يلاحظ أنه بجانب التدخلات الاستعمارية، وظهور فواعل جديدة بالمنطقة، فقد أدت التوترات الناجمة عن النزاعات الحدودية في المنطقة هي الأخرى إلى تأجيج الصراع في المنطقة؛ حيث أدت الطبيعة غير المحددة للحدود في القرن الأفريقي

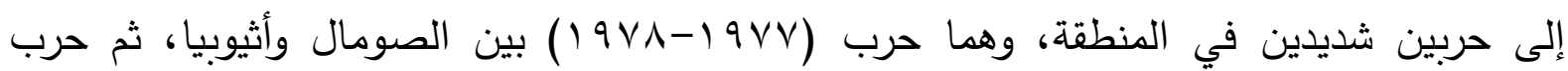
(991 ا - . . . ץ) بين إريتريا وأثيوبيا، مما نتج عنهما نزوح كبير داخل البلدان وعبر الحدود مما جعل القرن الأفريقي أحد المناطق الرئيسية المولده لهؤلاء اللاجئين('1). كما كانت للمناطق الحدودية المحددة بين السودان وأثيوبيا والسودان وأوغندا والصومال وأثيوبيا في حالة ما يطلق عليه: "الأزمات المتقطعة"

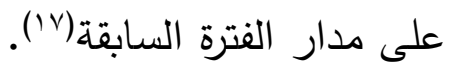

ويمكن القول بأن الارس الرئيسي الذي يمكن الاستفادة منه في شأن الطبيعة الجيوسياسية وديناميات الصراع في القرن الأفريقي يتلخص في النقاط التالية: ا-ساعدت العوامل السياسية والاقتصادية والإقليمية والتاريخية والأيديولوجية والبيئية على إحداث التوترات بين دول المنطقة مما ولد نوع من العداء والتنافس وعدم الثقة المتبادل بين دول المنطقة؛ مما ساعد في ظهور ما يسمى:"بالدعم الخارجي عبر الحدود" لبعض الحركات بهدف إحداث بعض

$$
\text { التوترات التي ينجم عنها صراعات محلية(^'). }
$$


ץ-ساعدت التدخلات الخارجية للقوى الفاعلة من خلال ظهور ما يسمى بـ:الفاعلين الجدد في المنطقة بكافة صورها في تشكيل وتصعيد الصراعات بين الدول مما أدى إلى زعزعة الاستقرار في الدول

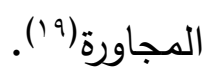

ب-إن إخفاق دول المنطقة في ظل الصرعات السابق ذكرها، خلقت نوع من التوترات فيما يتعلق بتوزيع القوة والعوائد الاقتصادية بين دول المنطقة(rr). ع-إن فشل دول منطقة القرن الأفريقي في إنشاء المشروعات الإنمائية وتحقيق التنمية ومعالجة المشكلات الخاصة بها، وعدم قدرتها على توسيع مؤسساتها أدى إلى الحروب الأهلية وظهور ما

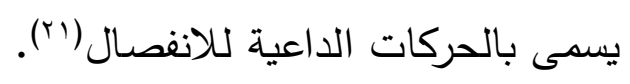

ه- أدى تكرار الصراع وعدم احتكار دول المنطقة لوسائل الردع إلى انتشار الصرعات المتعددة بكافة

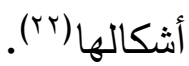

ج-في كثير من الأحيان تؤدى التوترات إلى صراعات داخلية ذات أثر مختلف وأبعاد متعددة، ولم يقتصر الأمر عند هذا الحد؛ بل قد تمتد إلى حروب مبررة، وأحياناً قد تؤثر هذه الصراعات الداخلية على دول القرن الأفريقي ككل؛ بحيث يكون أثارها ممتد خارج حدود الدولة(r؟). ويستخلص مما سبق مدى ارتباط الطبيعة الجيوسياسية وديناميات الصراع في القرن الأفريقي بمسارات معينة مرتبطة بتواجد الفواعل الإقليم والدولية في المنطقة، وتتضح هذه المسارات من وجود توترات بين دول المنطقة تقودنا إلى تعريف القضية الرئيسية للدراسة، وهذا ما سوف يتضح من خلال التحليلات القادمة.

\section{المحور الثاني: القرن الأقريقي في الحسابات الجيوسياسية الإقليمية والدولية} شكل التنافس خلال الحرب الباردة بين القطبين للسيطرة والتحكم بمنطقة القرن الأفريقي، نوعاً من سباق النفوذ الدولي بالمنطقة؛ حيث تبادل الطرفين الأدوار وتعاظم التنافس بينهما، وكان الرهان الأمريكي على احتواء المد السوفيتي؛ فيما راهن الأخير على التركيز على التواجد في المنطقة في كل من الصومال وأثيوبيا، وهذا ما أعتبر من قبل الولايات المتحدة تقليصاً، وتقييداً لنفوذها الاستراتيجي بالمنطقة، وأكد هذا

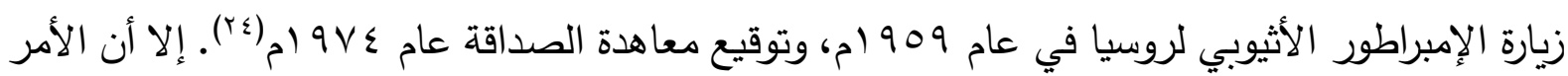
قد اختلف إبان انتهاء الحرب الباردة؛ حيث دخلت المنطقة في تحولات محورية، فبالرغم من تقلص الدور 
السوفيتي في المنطقة ظل التواجد الولايات المتحدة بالمنطقة محورياً؛ حيث عززت تواجدها بالمنطقة من

خلال التعاون الأمني مع دول المنطقة عبر تقديم المساعدات اللوجستية والتمويلية لدول المنطقة(ror). ووفقاً للمعطيات السابقة يتضح جلياً أهمية منطقة القرن الأفريقي التي أصبحت تشكل اهتماماً مركزياً في أجندة صانعي السياسات الخارجية لدى القوى الفاعلة والقوى الصاعدة الأخرى. وعليه فقد بدأت القوى الإقليمية والدولية الفاعلة في العمل على إعادة صياغة منطقة القرن الأفريقي من أجل تحقيق الهيمنة والنفوذ في هذه المنطقة عبر صياغة مفهوم (القرن الأفريقي الكبير) ليعبر عن المصالح السياسية والاقتصادية والأمنية والإستراتيجية في مناطق شمال وشرق أفريقيا ومنطقة البحيرات العظمى. ويتضح ذلك من خلال تحليل جيوسياسية القرن الأفريقي في الحسابات الإستراتيجية الدولية والإقليمية على النحو التالي:

أولاً:القرن الأفريقي في الحسابات الجيوسياسية الدولية(الولايات المتحدة الأمريكية، روسيا، الصين): يترتب على الأهمية الدولية للقرن الأفريقي، أن أصبحت القوى الفاعلة الدولية تهتم اهتماماً كبيراً بمراقبة الأحداث السياسية في المنطقة، تلك الأحداث التي تخدم أهدافهم في النفاذ إلى الشرق الأفريقي، والتي ساهمت بالفعل في إدخال القارة الأفريقية في لعبة التوازن الدولي، وعلى مقتضى تلك الأهداف يتم تناول القرن الأفريقي في الحسابات الجيوسياسية الدولية على النحو التالي: 1-القرن الأفريقي في الحسابات الجيوسياسية الأمريكية: لقد مثلت أحداث الحادي عشر من سبتمبر ا . . بم، دوراً هاماً في تشكيل العلاقات الدولية، وأضافت أبعاداً جديدة على المجتمعات في العالم كافة؛ حيث أضفت تلك الحادثة تأثيراً مباشرا،ةً ومهماً في البنية العامة للمجتمع، والتفكير الاستراتيجي الأمريكي، وبذلك أصبحت الرؤية الأمريكية للبيئة الدولية مصحوبة برؤية خاصة؛ قامت على إثرها الإدارة الأمريكية بإدخال مفهومين جديدين في التفكير الاستراتيجي الأمريكي، تمثل المفهوم الأول في: أن التهديد الأهم لأمن الولايات المتحدة الأمريكية يقع على نقطة التقاطع بين الراديكالية والتقدم التكنولوجي، سواء أكانت الراديكالية سمة لدولة أم لفئة سياسية، وجاء المفهوم الثاني متمثلاً في: الضربات الاستباقية للولايات المتحدة الأمريكية؛ فهي لن تنتظر حتى تتعرض للهجوم لكي تقوم بالرد عليه، وإنما سيكون عليها أن تبادر بالهجوم بمجرد إدراكها للتهديد(؟r)، وجاءت أهمية القرن الأفريقي في الحسابات الجيوسياسية الأمريكية من خلال مجموعة من الديناميات تمثلت في: 
أ-الايناميات السياسية: وجاءت من منطلق العلاقات الثنائية الجيدة مع دول القرن الأفريقي بصفة عامة وإن كانت على درجات متفاوتة، تتوقف على الظروف والأوضاع السياسية لكل بلد على حده؛ إضافة إلى تغير المصالح الآنية بتقلب المناخ السياسي، ومقدار حجم التهديدات الناجمة عن مشكلات الفقر وضعف الدولة على نحو يجعلها مهيأة للحركات الإرهابية وظهور فواعل غير رسمية

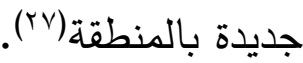
ويوضـح الجدول (1) تطور العلاقات الأمريكية الدبلوماسية مع بعض دول القرن الأفريقي على النحو التالي (*)

\begin{tabular}{|c|c|c|}
\hline تاريخ العلاقًات الدبلوماعبِة & المولية & م \\
\hline 19.4 & أثنيوبيا & -1 \\
\hline 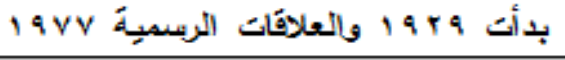 & جيبوتَى & $-r$ \\
\hline $19 \leqslant 5$ & إريتريا & -4 \\
\hline 1907 & المبودان & $-\varepsilon$ \\
\hline 197. & الصوماJ & -0 \\
\hline
\end{tabular}

ب-الايناميات الاقتصادية: وتمثلت في الاستفادة من الأوضاع الاقتصادية المتردية لاقتصاديات دول القرن الأفريقي نتيجة لمعاناة معظم دول القارة من عدم الاستقرار السياسي؛ وجاء ذلك بهدف حماية مصادر الإنتاج النفطي، ورصد تحركات أي منافس حقيقي أو محتمل للولايات المتحدة في القرن الأفريقي من شأن تحركاته أن تضر بأمن الطاقة الأمريكية. لضمان تدفق تصدير النفط الأفريقي ووصوله إلى الولايات المتحدة الأمريكية دون أي مشكلات، مما يساعد على التقليل من الاعتماد على نفط الشرق الأوسط في إطار سياسة تتوع الواردات الجغرافية وأنواع الطاقة؛ حيث تعُد أفريقيا عنصر رئيسي في

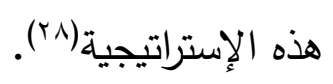

كما استفادة أيضاً من طبيعة الأوضاع الإقليمية، وعلى وجه الخصوص الخلافات بين دول المنطقة، والتي جعلت البعض منها يسعى للحصول على تسهيلات عسكرية؛؛ مما انعكس على طبيعة التوازنات ونمط العلاقات الإقليمية بين دول منطقة القرن الأفريقي. كما تم تركيز دورها في تعزيز أمن وبناء قدرات دول المنطقة الحليفة للولايات المتحدة، وتم ذلك من خلال تكثيف دور وعمل "الوكالة الأمريكية للتنمية الدولية" والفريق الدبلوماسي للولايات المتحدة التابعة "لقوة المهام المشتركة"(") في منطقة "القرن الأفريقي"، بهدف تعزيز قدرات تلك الدول، الأمنية والاقتصادية والعسكرية لكي تساعد الولايات المتحدة 
في حماية مصالحها الاقتصادية في المنطقة؛ حيث جاءت الأهداف المعلنة من تكوين "قوة المهام المشتركة" في المنطقة متمثلة في زيادة قدرات الدول الشريكة في شرق أفريقيا على الحفاظ على بيئة مستقرة، وتوفير درجة من التقدم الاقتصادي والاجتماعي لمواطنيها للإسهام في التتمية الاقتصادية العالمية، وتحقيق نوع من الاستقرار على المدى الطويل في المنطقة، بما يضمن تحقيق مصالحها بالمنطقة(9). كما طرحت عدة مبادرات لزيادة وتشجيع التجارة بينها وبين دول القرن الأفريقي بصفة خاصة والقارة الأفريقية بصفة عامة، جاء منها على سبيل المثال مشروع النمو والفرص الأفريقي "الاغو"؛ من خلال الجولة التي قامت بها "الرئاسة الأمريكية" في 99 (م، والتي شملت ست دول أفريقية منها: دولتان من دول القرن الأفريقي هما: أوغندا ورواندا، وقد ركزت الزيارة في الجوانب الاقتصادية بالعلاقات الأمريكية -الأفريقية؛ حيث تقرر خلالها إلغاء • ب مليون دولار من الديون المستحقة للولايات المتحدة الأمريكية على بعض الدول الأفريقية، وكذلك تخصيص · ب مليون دولار لبرنامج تتمية التجارة والاستثمارات، وتم

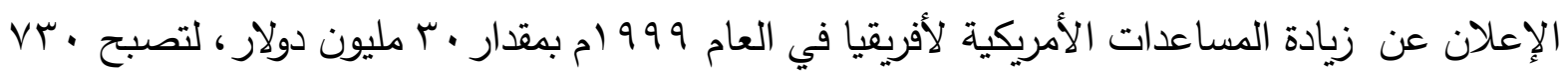
مليون دولار ، ويوضح الجدول (r) حجم الصادرات والواردات الأمريكية من وإلى دول القرن الأفريقي؛ مما يرسخ الهدف من التوجهات في القرن الأفريقي والتي تمثلت في: السيطرة على المخزونات الأفريقية من النفط والحصول علية بأسعار منخفضة، ولاسيما بعد الاكتشافات الجديدة، وتتوع مصادر الطاقة الخاصة بها، والحد من المد الصيني في القارة الأفريقية، والعمل على استمرار الصراعات وهذا ما أكدته كافة

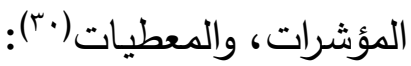

جدول (ץ) حجم الصادرات والواردات الأمريكية من وإلى دول القرن الأفريقي("):

\begin{tabular}{|c|c|c|c|c|c|c|}
\hline \multirow[t]{2}{*}{ التَربَبِ } & \multicolumn{2}{|c|}{ المواربات (بالمليونون دولار) } & \multirow[t]{2}{*}{ التَربتِب } & \multicolumn{2}{|c|}{ الصنادرات (بالمليونِ دولار) } & \multirow[t]{2}{*}{ الدولة } \\
\hline & $r . r .-r .19$ & $r .19-r .11$ & & $r . r .-r .19$ & $r .19-r .11$ & \\
\hline $\operatorname{lv\varepsilon }$ & $1,,$. & $1 \varepsilon$, & rit & $r, \cdot$ & $1 \varepsilon$, & بورندى \\
\hline $1 \leqslant 9$ & r, & $\varepsilon \wedge$, & דיזו & 101,0 & 11,0 & جبيوتئي \\
\hline rth & بـ ا ألف & مrvo ألف & 191 & $1 \varepsilon$, & $\wedge, \cdot$ & إريتريا \\
\hline$\wedge \wedge$ & ovr, & $\varepsilon \varepsilon 0,$. & V V & ا & هو م, املِيار & أثِنيويِيا \\
\hline$\wedge \mathrm{T}$ & $7 \%$, & $\nabla \varepsilon r, \cdot$ & 1.9 & $\varepsilon \cdot 1$, & ro,. & 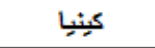 \\
\hline r & $\varepsilon \uparrow$, & $\uparrow \wedge, \cdot$ & 119 & 19,0 & ro,. & روندا \\
\hline ris & ז १ ألف & $1, r \cdot \Lambda$ & $1 \varepsilon v$ & $1.0,0$ & $\varepsilon q, \cdot$ & الصومال \\
\hline 101 & ri, & $10,$. & $17 \varepsilon$ & $v \varepsilon, *$ & $q_{0, *}$ & التسودان \\
\hline 119 & Ir.,. & $9 \vee,$. & 111 & 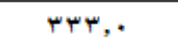 & 9q & تيزائيا \\
\hline $1 \pi v$ & $\wedge \cdot, \cdot$ & $\uparrow, \cdot$ & 10. & $1 . \varepsilon$, & $\wedge \vee, \cdot$ & أوغثدا \\
\hline سזו & $\pi, \cdot$ & - & 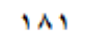 & $\wedge, \cdot$ & - & جنوب السودان \\
\hline
\end{tabular}


ج-الايناميات الأمنية: من المنطلق الأمني، اعتمدت الخطط الأمريكية المستقبلية إلى جعل موضوع "الإرهاب" كأحد محددات التوجه الأمني، وجاء من الأهداف الإستراتيجية لها متمثلاً في إعادة صياغة التوازن الاستراتيجي في العالم؛ بما يضمن لها السيطرة على أوسع رقعة ممكنة، وعليه احتلت منطقة القرن الأفريقي مكانة مهمة في الفكر الاستراتيجي الأمريكي الخاص بمحاربة الإرهاب؛ حيث يمثل القرن الأفريقي بوجه خاص منطقة تتكاثر فيها التهديدات للمصالح الأمريكية ولحلفائها؛ لما تعانيه المنطقة من عدم استقرار في الأوضاع السياسية - كما سبق توضيحه من قبل(اب). ولهذا اتجهت الولايات المتحدة الأمريكية لمواجهة التحديات الأمنية التي تعترض المصالح الأمريكية في المنطقة من خلال تعزيز تعاونها الأمني مع دول المنطقة عن طريق ثلاثة مهام رئيسية تمثلت أولاها: في العمل على كسب الود من خلال تقديم المساعدات الإنسانية، وثانيها: منح الدراسات العسكرية التي عقدتها مع (بورندى، وجيبوتى، وإريتريا، وأثيوبيا، وكينيا، وتنزانيا، وأخيراً أوغندا) والثالثة تدريبهم لمواجهة الإرهاب(rr). وخلاصة القول في شأن ما سبق - فإن المتمعن في الأحداث يلاحظ أن القرن الأفريقي لم يشكل أهمية كبيرة للولايات المتحدة بالنسبة للإستراتيجية الدولية خلال فترة الستينيات، ويرجع السبب في ذلك إلى أن نفوذ الاتحاد السوفيتي في القرن الأفريقي لم يكن بالثئ المقلق للولايات المتحدة إلا أن الأمر قد اختلف بعد انسحاب البرتغال من المستعمرات الأفريقية ابتداء من عام ؟ \و ام؛ حيث سعت الولايات المتحدة لمحاربة النفوذ السوفيتي بعد أن زاد خلال تلك الفترة(r). مما يفسر تحرك الولايات المتحدة تجاه القارة الأفريقية عامة، والقرن الأفريقي خاصة؛ حيث يُرجع التحرك إلى مجموعة من العوامل بالإضافة إلى العوامل السالف ذكرها والتي تحاول الولايات المتحدة تحقيقها بغطاء مكافحة

$$
\text { الإرهاب والتي نوردها في الآتي (عَ): }
$$

• السيطرة على منابع النفط في القارة، وما يؤكد ذلك ويرسخه ويعطى مؤشراً عليه، اشتداد الصراع بين الولايات المتحدة والصين على النفط في دول "القرن الأفريقي". " مواجهة تصاعد النفوذ الروسي، والصيني في القارة وعلى وجه الخصوص الصراع على موارد الطاقة. • محاصرة النفوذ السياسي والاقتصادي الأوروبي المتزايد في بعض مناطق القارة الأفريقية، وما يؤكد ذلك، التنافس الحادث بين الولايات المتحدة وفرنسا في منطقة الشرق، والغرب الأفريقي 
حالياً، والمناطق التي تقع في إطار نطاق مسؤولية "قوة المهام المشتركة" لمنطقة القرن الأفريقي،

$$
\text { وهى (السودان، وأثيوبيا، وإريتريا، وجيبوتى، والصومال، وكينيا، وجزر سيشيل). }
$$

\section{ب-القرن الأفريقي في الحسابات الجيوسياسية الروسية:}

أوضحت وثيقة السياسة الخارجية التي صدرت في عام ^ . . بم، والتي وقعها الرئيس السابق "ديمترى

ميدفيديف" المكانه الأفريقية عامة، والقرن الأفريقي خاصة في الحسابات الروسية؛ حيث احتلت أفريقيا المرتبة التاسعة بين قائمة المناطق العشرة الأكثر أهمية بالنسبة للمصالح الروسية، في حين أن الوثيقة الدحرة في 10 • بم تثير إلى أن الدولة الروسية ستتوسع في علاقاتها مع دول قارة أفريقيا في مختلف المجالات؛ سواء على المستوى الثنائي، أو مستوى السياسية الخارجية للاتحاد الروسي التي وقعها الرئيس بوتين في عام 17 • بم، وذلك من خلال تحسين الحوار السياسي، وتكثيف التعاون الثامل بكافة صورة السياسية والاقتصادية والعسكرية، وكذلك التعاون في الثأن الإنساني والتعليمي، بما يخدم المصالح

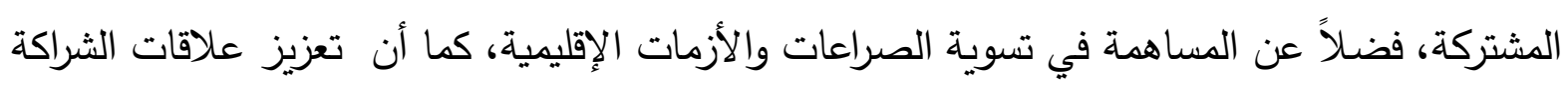
مع الاتحاد الأفريقي هو بمثابة عنصر هام في السياسة الروسية تجاه أفريقيا(º). ومن ثَم أضحت أفريقيا هدفاً بارزًا في إطار تجديد روسيا لإستراتيجيتها الدولية، وعلى وجه الخصوص في ظل تعاظم تأثيرها على الصعيد الدولي؛ إذ لم تكن منطقة القرن الأفريقي ضمن اهتمامات السياسة الروسية كمنطقة هامة على الصعيدين الاستراتيجي والجيوسياسى في الفترة التي أعقبت انهيار الاتحاد السوفيتي؛ نتيجة لفقدان الاهتمام الروسي للقارة ككل خلال تلك السنوات. ولم تقتصر نظرة الروس للقرن الأفريقي على أنها منطقة تتمتع بالعديد من الصراعات الإقليمية فقط؛ بل امتدت لتثمل تحديات أمنية كبرى تمثلت في ظهور فواعل جديدة بالمنطقة، وانتثار ظاهرة الإرهاب والجريمة العابرة للحدود، والجرائم المنظمة؛ مما دفع الاتحاد الروسي لزيادة النفقات العسكرية للاتحاد الروسي وهذا ما تفسره الرؤية الروسية(بَ). ونستخلص مما سبق أن التوجه الروسي للمنطقة في ظل التطورات الدينامية التي تشهدها المنطقة سيترتب عليه تغيير المشهد الجيوسياسى، وإعادة ما يطلق علية "هندسة العلاقات الدولية" في القرن الأفريقي مما جعل روسيا تقدم نفسها لدول المنطقة على أنها شريك أساسي خلال المرحلة الراهنه. ويمكن القول بأن التوجه الروسي شأنه شأن التوجه الأمريكي يتمثل في مجموعة من الديناميات تأتى على النحو التالي: أ-الايناميات السياسية: حيث تسعى روسيا إلى تجديد العلاقات مع دول القرن الأفريقي، وتعزيز تأثيرها

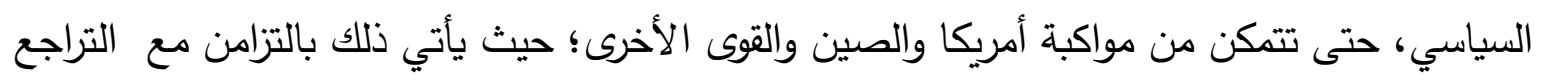


الأمريكي بعض الثيء في المنطقة، وفى ظل الانتشار السريع للصين في المنطقة؛ فضلاً عن حرصها على تقديم الدعم للاتحاد الأفريقي من أجل إيجاد تمويل مستدام لعمليات السلم والأمن في المنطقة(rvr). كما أن المخاوف الروسية من حدوث عزله دولية ودبلوماسية من الدول الغربية نتيجة لموقفها في سوريا وأوكرانيا وبعض الدول الأخرى دفعها إلى البحث عن حلفاء جدد، ومناطق نفوذ لها بخلاف الشرق الأوسط كمنطقة القرن الأفريقي لمواجهة هذه العزله وعلى وجه الخصوص مع الدول التي تخضع للعقوبات

$$
\text { من الولايات المتحدة والدول الغربية(^^م). }
$$

ب-الايناميات الاقتصادية: مع العزم على العودة إلى المنطقة أصبحت العلاقات بين روسيا وأفريقيا أكثر ديناميكية يعززها الاهتمام بهذا النمو تزايد مصالح الصين والهند والبرازيل، ولاسيما في الدول الأفريقية الموحدة بهدف الوصول الآمن إلى الموارد الطبيعية واحتياطيات الطاقة، وبفضل مواردها الطبيعية، أغلقت روسيا ديونها لفترة قصيرة، وأنقذت نفسها من مراقبة "صندوق النقد الدولي". وهكذا، بدأت روسيا سياسة خارجية جديدة أكثر استقلالية مرة أخرى(q). هذا بالإضافة لمشاركة روسيا في مجموعة الثمانى مما يمنحها دعم وثقة أكبر من منطلق اشتراكها في مجموعة ذات قوة اقتصادية مميزة(•؛). فمنذ بداية القرن الجديد، تم إجراء عدة زيارات رسمية لروسيا من قبل قادة ووزراء خارجية الدول الأفريقية بما في ذلك كينيا، إريتريا وإثيوبيا، وفى المقابل قام وزراء روس، ومسئولون آخرون بزيارة بلدان أفريقية. وعقدت

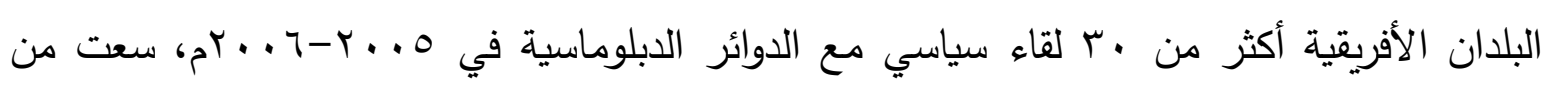
خلالها القيادة الروسية التعاون مع المؤسسات الإقليمية الأفريقية، وجاء منها الاتحاد الأفريقي في عام 0 . . ب ، وفى هذا الإطار تم أيضاً اعتماد سفير الاتحاد الروسي لدى أثيوبيا في مفوضية الاتحاد

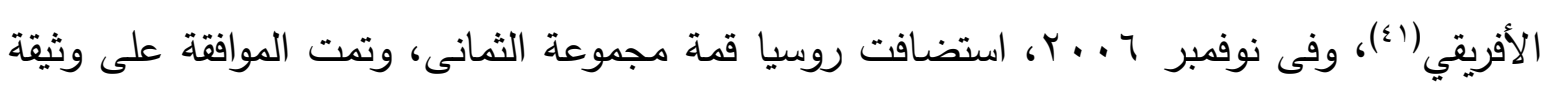
القمة حول "تحديث أفريقيا"، هذا بالإضافة إلى الإشارة إلى المشاكل الأفريقية أيضًا في الوثائق الأساسية والنهائية للقعة؛ حيث تم تنمية العلاقات التجارية والاقتصادية، وتعزيز التجارة الثنائية مع دول المنطقة في مختلف القطاعات. وفتح منافذ جديدة، يمكن من خلالها تصريف المنتجات والخدمات والتقنيات الروسية(ז^)، والاستفادة من ثروات المنطقة من الموارد الطبيعية(זء)، وفى سبيل ذلك أطلقت روسيا في عام 10 • ب م المنتدى الروسي الأفريقي. ج-الايناميات الأمنية: تمثلت في الرغبة في المشاركة في احتواء خطر الفواعل الغير رسمية الجديدة؛ بما لا يهدد المصالح الروسية في المنطقة، ويحجب عنها خطر هذه الفواعل في أن يمتد إليها أو للمناطق 
المجاورة لها(؛؛). والمساهمة في تأمين المصالح الروسية في البحر الأحمر ، والمشاركة في حماية مضيق باب المندب، فضلاً عن القرب من الأزمات الإقليمية في منطقة الشرق الأوسط(؛؛)، والعمل على استعادة النفوذ الروسي في ظل التواجد الدولي والتتافس الإقليمي في منطقة القرن الأفريقي(؟ء). ويتجلى السعي الروسي إلى التغلغل في منطقة القرن الأفريقي من خلال تعزيز علاقاتها السياسية والاقتصادية مع دول المنطقة فعلى المستوى الأثيوبي مثل قطاع الطاقة مدخلاً هاماً لروسيا لفتح المجال أمام شراكة قوية بينها وبين أثيوبيا؛ بحيث أصبحت الأخيرة البوابة الروسية لأفريقيا؛ حيث تعمل شركة "Rosatom" في العديد من دول المنطقة(اء)، وعلى المستوى الإريترى مثلت أسمرة مدخلاً هاماً لموسكو لإيجاد موطئ قدم في القرن الأفريقي؛ حيث تطورت العلاقات الثنائية خلال السنوات الماضية، على المستوى السياسي والاقتصادي، امتدت لتشمل بناء قدرات إريتريا العسكرية، فضلاً عن إنشاء أسطول روسي في البحر الأحمر لحماية مصالحها، كما قامت بتدريبات بحرية مشتركة(^§). وعلى المستوى الصومالي رأت روسيا فيها موردًا هاماً للعديد من الموارد الطبيعية غير المستغلة؛ مثل النفط والغاز واليورانيوم، بالإضافة إلى حاجة الصومال لشريك استراتيجي يسهم في إعادة البناء في ظل عدم الاستقرار الذي تتمتع به ؛ حيث سعت الصومال للاستفادة من الخبرة العسكرية الروسية في محاربة الإرهاب، وعلى المستوى السوداني، تمتعت روسيا بعلاقات ثنائية على المستوى السياسي والاقتصادي والعسكري؛ حيث كانت روسيا داعماً رئيسياً للسودان على المستوى العسكري هذا في ظل توتر العلاقات الأمريكية السودانية(9٪). وفى جنوب السودان اعتمد الرئيس" سلفاكير ميارديت" على روسيا والصين في الوقوف إلى جانبها ضد العقوبات الأمريكية على بلاده، وحظر الأسلحة من مجلس الأمن الدولي؛ بسبب الصراعات والحرب الدائرة في البلاد منذ منتصف ديسمبر r ا ـ rم، ومن ثم رغبت جنوب السودان في تعزيز العلاقات مع روسيا من أجل التوازن في علاقاتها مع الولايات المتحدة الأمريكية(•). ب-القرن الأفريقي في الحسابات الجيوسياسية الصينية: لم يقتصر التوجه الدولي في القرن الأفريقي على الولايات المتحدة؛ بل امتد ليشمل قوى صاعدة أخرى منافسة لهما متمثلة في الصين التي تسعى إلى تطويق القوى الأخرى بسياج أمنى؛ إذ دخلت منطقة القرن الأفريقي حيز السباق على الهيمنة نظراً لاعتبارها أحد أهم، وأغنى أقاليم العالم الجيوسياسية؛ حيث مثل التوجه الصيني وحضوره في القرن الأفريقي أهمية كبرى ليس على مستوى القرن الأفريقي فقط؛ بل امتد ليشمل القارة ككل؛ حيث يعود التواجد الصيني في القارة الأفريقية إلى 
التقدم الذي شهدنه السياسة الخارجية الصينية، وما صاحبها من تصاعد دور ونفوذ الصين كقوة عالمية إبان تفكك الاتحاد السوفيتي، وانفراد الولايات المتحدة بقيادة العالم في ظل سياسة الهيمنة والقطب الواحد؛ حيث اتجهت الصين لتطبيق سياسة التوسع، والعمل على التوازن الاستراتيجي مع الولايات المتحدة، والبحث هي الأخرى عن وجود أحلاف لها لإثبات تواجدها ونفوذها بهدف تأمين مصادر النفط. ولم يقف الأمر عند هذا الحد؛ بل سعت الصين لأن تكون شريك قوى ومنافس للولايات المتحدة وفرنسا في القارة الأفريقية؛ حيث تضاعفت التجارة بين الصين وأفريقيا فقد ارتفعت إلى بس٪

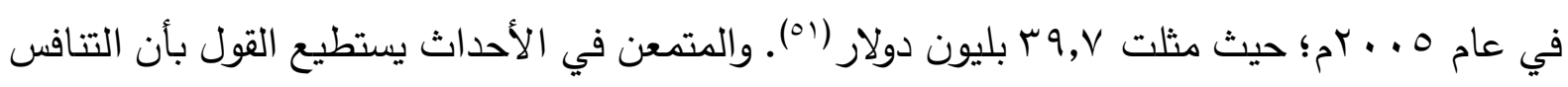
الصيني الأمريكي على القرن الأفريقي يحمل في ظاهرة صبغة اقتصادية، فالصين تمتلك منطقة لوجيستية هامة في "جيبوتى"، إلا أن ذلك يختلف عن الرؤية الخاصة بالولايات المتحدة؛ حيث ترى أن التواجد الصيني يحمل في طياته الصبغة الأمنية؛ حيث يشكل هذا التمدد خصماً من رصيد الولايات المتحدة في منطقة القرن الأفريقي(ro). ويلاحظ أن التوجه الصيني نحو القرن الأفريقي شأنه شأن التوجه الأمريكي والروسي يتمثل في مجموعة من الأبعاد الرئيسة تمثلت في: أ-الايناميات السياسية: لقد أصبحت المنطقة بعد استقلال دولها تتمتع بالتنافس بين العديد من القوى الدولية؛ مما يؤدى في الكثير من الأحيان إلى الوقوع تحت التأثير السياسي الغير مباشر لتلك القوى، وهذا هو مفهوم "التبعية الجديد" الذي تحاول دول المنطقة خاصة وأفريقيا عامة الإفلات من قبضته والذي يعد في صورته هذه أخطر بكثير من الاستعمار القديم(هo). ولقد اتسمت العلاقات الصينية - الأفريقية بالقدم فدعم الصين لحركات التحرر الأفريقية التي بدأت في خمسينيات القرن الماضي تعتبر الركيزة الرئيسية التي انبثقت منها العلاقات، وتمثل الأيديولوجية الاشتراكية السمة السائدة في العلاقات الصينية الأفريقية(؟). إلا أن الأمر اختلف نتيجة للتغيرات الدولية مع بداية تسعينيات القرن الماضي والتطور الذي عرفته الصين؛ حيث تحولت علاقاتها بدول القرن الأفريقي خاصة وبأفريقيا عامة من الارتكاز على العوامل الأيديولوجية، ومساندة النظم ذات الطابع الثيوعي إلى إقامة علاقات يغلب عليها طابع المصالح، والمنافع المتبادلة وربما أصبح العنصر الأيديولوجي الوحيد والذي ظل قائماً في علاقة الصين بدول القارة هو مبدأ "صين واحدة"(00)، 
ويوضح الجدول (r) العلاقات الدبلوماسية الصينية مع دول القرن الأفريقي(†ه):

\begin{tabular}{|c|c|c|c|}
\hline تاريتخ التعلاقات الدبلوماسبية & تاربِخ الاستقَلدل & الدولة & م \\
\hline 1901 & 1907 & الهبحداث & -1 \\
\hline 197 & 197. & الصومسا & $-r$ \\
\hline 1971 & 1971 & تيزانيا & 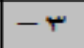 \\
\hline 197 & שר & كيثيا & $-\varepsilon$ \\
\hline $197 \leq$ & 1975 & أوغثدا & -0 \\
\hline $19 \vee \cdot$ & - & أثيويبيا & -7 \\
\hline 1981 & 197 & טفند1 & $-\vee$ \\
\hline $197 \leq$ & 197 & بورثب & $-\wedge$ \\
\hline $19 \vee 9$ & $19 v 8$ & جيبوتَت & -9 \\
\hline 1990 & 1994 & إريتَريا & -1 \\
\hline$r+1$ & +1 & جنوب الهبودات & -1 \\
\hline
\end{tabular}

ب-الديناميات الاقتصادية: جاءت التوجهات الاقتصادية الصينية تجاه دول القرن الأفريقي متمثلة في فتح أسواق جديدة في أفريقيا فبعد أن انتزعت الصين نصف الأسواق الأفريقية منذ عام .... بم أصبحت ثاني أكبر شركاء القارة الأفريقية في عام • 1 • rم بعد الولايات المتحدة الأمريكية، وقبل فرنسا التي كانت تشغل مكانه كبيرة في اقتصاديات القارة، كما تمثل هذا البعد فيما قامت به الصين من تأسيس العديد من المؤسسات الاقتصادية ذات الطابع التعاونى المُتميز لتعزيز العلاقات على جميع الأصعدة بينها وبين دول المنطقة، إذ كانت تهدف تلك المؤسسات إلى ترسيخ العلاقة القوية بين الصين ودول القارة الأفريقية عموماً ودول منطقة القرن الأفريقي بشكل خاص، ومن أمثلة تلك المؤسسات "

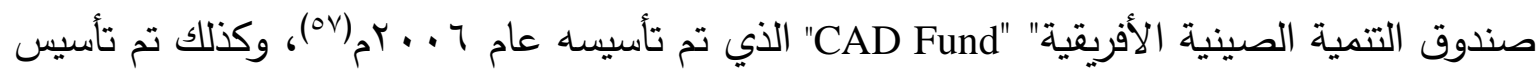
منتدى التعاون بين بكين والقارة الأفريقية "Focac " ليكون مُكملاً في عمله لصندوق التتمية سالف الذكر ويهدف هذا المُنتدى إلى المساواة والمنفعة المُتبادلة والتشاور المُتكافئ وتعزيز التفاهم وتوسيع التوافق المُشترك وتقوية الصداقة ودفع التعاون، ويضم المُنتدى في عضويته الصين وساه دولة أفريقية تُقيم علاقات دبلوماسية مع الصين ومفوضية الاتحاد الأفريقي بهدف التنسيق والتعاون المُشترك بين الدول الأفريقية والصين؛ إذ كان حجم التبادل التجاري الصيني الأفريقي في بداية التأسيس هو • ( مليار دولاز ، وبعدها بأربعة أعوام تجاوز حجم التبادل بين الجانبين • ـ مليار دولار (ㅅ)، 
ويوضت الجدول (ع ) حجم التعاملات الاقتصادية الصينية(ه):؛

\begin{tabular}{|c|c|c|}
\hline حجم الانستئمارات بالمليوت دولار & الثولية & التربكِب \\
\hline $10, v, \cdots \varepsilon$ & السبود ات & -1 \\
\hline$\vee \vee \vee, \wedge \leq$ & أثخيويبيا & $-\leftarrow$ \\
\hline$v 17, \varepsilon 7$ & تيزانيا & 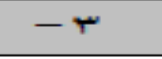 \\
\hline 70,9 & كيثيا & $-\varepsilon$ \\
\hline 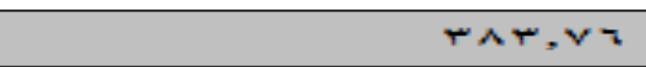 & أوغتد1 & -0 \\
\hline $1 \cdot \varepsilon, \infty 0$ & إربيتربيا & -7 \\
\hline שיו,מיט & טגتז1 & $-v$ \\
\hline 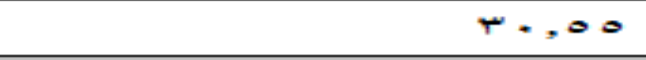 & جيبوتي & $-\wedge$ \\
\hline$r y, \leqslant v$ & جثقب السبحداث & -9 \\
\hline$a, v a$ & بوربتد & $-1 \cdot$ \\
\hline- & الصومس M & -1, \\
\hline
\end{tabular}

ج-الايناميات الأمنية: خلف الحراك الشعبي في بعض الدول الأفريقية وضعاً جديداً في المنطقة وبالرغم من أنه لا يزال من المبكر تحديد مدى تأثير هذه الأحداث في مصالح القوى الأجنبية، إلا أن ذلك لا يحول دون تبين بعض السيناريوهات الأولية إن صح التعبير ، وعلى وجه الخصوص ما يتعلق بسلوك القوى الكبرى كالصين، فمن أكثر الأمور الكاشفة لتحول السياسة الصينية تجاه الأحداث الأفريقية هو موقفها من بعض القضايا والأزمات الأفريقية، ومنها على سبيل المثال الأزمة الليبية، فهي لم تستخدم حق الفيتو كما كان متوقعاً ضد القوى الغربية فيما يخص الثأن الليبي؛ الأمر الذي كلف الاقتصاد الصيني خسائر كبيرة؛ وجاء ذلك على خلاف الموقف الصيني تجاه العقوبات السورية؛ حيث استخدمت الصين حق الفيتو ضد قرار فرض العقوبات في مجلس الأمن، وقد يرجع السبب في ذلك إلى: التأثر بالموقف الروسي المدافع عن المصالح الجيواستراتيجية هنالك، ومن وجه نظر أخرى المحافظة على العلاقات الصينية الروسية وعدم المخاطرة بفقدان الدعم الروسي لها، والذي قد يكون داعماً لها في المستقبل. كما يكشف هذا الموقف الصيني أحد ردود الأفعال المباشرة على ما قامت به الولايات المتحدة من الإعلان عن التحول في إستراتيجيتها نحو منطقة المحيط الهادي الآسيوي، وهى الإستراتيجية التي تم التعبير عنها من قبل الرئاسة الأمريكية بأنها "مراجعة دفاعية تقوم على تركيز القوات الأمريكية في المحيط الهادي" والتي تتم عن توتر العلاقات بين الطرفين في هذه المنطقة نظراً إلى إدراك الصين أن الولايات المتحدة تقوم بما أطلق عليه بعض الباحثين "هندسة الجيوسياسية Geopolitical Engineering" وهو ما جعل الصين ترد في مناطق 
أخرى قد شكلت الأزمة السورية، والقرن الأفريقي فرصة لبعض هذا الرد(·؟). ففي خطوة جديدة لتؤكد التحول الاستراتيجي في السياسة الخارجية الصينية وتزايد الأهمية الجيوسياسية للقرن الأفريقي في هذه السياسة، أعلنت الخارجية الصينية في يناير 1 ا • بم التوصل لاتفاق مع جيبوتى لبناء قاعدة عسكريـة بحرية صينية، ستعد أول قاعدة عسكريـة تمتلكها الصين في الخارج، وهناك أيضاً مؤشرات عن توجه الصين لبناء قواعد في كل من السيشل، وموريشيوس وهو ما يعنى تصاعد الانخراط الصيني في القضايا الأمنية الأفريقية مستقبلاً (1).

\section{ثانياً: القرن الأفريقي في الحسابات الجيوسياسية الإقليمية (إيرلن، تركيا، إسرائيل):} بعد أن أصبح القرن الأفريقي يشكل أحد المجالات الجيوسياسية التي أثارت اهتمام القوى الفاعلية على المستوى الدولي، والتي عكست بدورها التغير في هيكل النظام ونمط التفاعلات الدولية وأدوار الأطراف والقوى الفاعلة فيه؛ كان من الضروري تتاول أهمية القرن الأفريقي في الحسابات الجيوسياسية للقوى الإقليمية بشيء من الإيجاز ؛ مما يعطى اعتبارات جديدة لمنطقة القرن الأفريقي يجعلها تخرج من دائرة التهيش إلى دائرة الاهتمام اللافت على المستوى الإقليمي هو الآخر . متمثلاً في تعاظم اهتمام القوى الإقليمية كإيران وتركيا وإسرائيل والتنافس الخليجي على المنطقة، ويرجع ذلك للعديد من الاعتبارات كالانفتاح على الموقع الجيواستراتيجى لممر البحر الأحمر في إطار مسعى التحكم في مسارات التجارة الدولية وتعدد مداخل

\section{1-القرن الأفريقي في الحسابات الجيوسياسية الإيرانية:}

جاءت المحاولات الإيرانية للانفتاح على أفريقيا بصفة عامة، ومنطقة القرن الأفريقي بصفة خاصة في إطار الإستراتيجية الإيرانية المعنية بتجاوز العقوبات المفروضة عليها إقليمياً، من خلال فتح آفاق جديدة لعلاقاتها مع دول القرن الأفريقي من منطلق إعادة النظر في سياستها الخارجية، والتي بدأت مع وصدل "هاشمى رافسنجانى" للرئاسة؛ حيث بدأت بزيارة للسودان في عام 9919 وأعقبتها زيارة لست دول هي "كينيا، وأوغندا، وتنزانيا، والسودان، وجنوب أفريقيا وزيمبابوى" في عام ד 9 ام؛ حيث مثلت هذه الزيارة قمت التعاون والمقاربة الإيرانية الأفريقية(זَ). وارتكزت إستراتيجية السياسة الخارجية للتوجه الإيراني على الاهتمام بالمنطقة باعتبارها المعبر الرئيسي لتأمين الانفتاح الإيراني على العالم الخارجي مع رؤية إستراتيجية بتوسيع نطاق حجم التفاعلات في ضوء المساعي لتفعيل التعاون الاقتصادي وتعزيز الروابط الاقتصادية والتجارية(rآ). 
وفى المقابل راهنت القوى الإقليمية لتعزيز وجودها بمنطقة القرن الأفريقي في إطار تدافعها مع

إيران، ومن خلال إستراتيجية الضغط والمساعدات استطاعت قطع العلاقات الإيرانية مع كلاً من جيبوتى والصومال وتعزيز تحلفها مع دول الخليج، وتقييد النفوذ والدور الإيراني بالمنطقة من خلال إنشاء قواعد عسكرية خليجية بالمنطقة؛ حيث بدأ صراع دول الخليج مع الجمهورية الإيرانية في عام 1 • بrم بتوقيع اتفاقية التعاون الخليجي الإريترى لتأمين وصول مجلس التعاون الخليجي إلى ميناء عصب، واستبعاد وصول طهران إليها(؟7).

ويمكن القول بأن التحركات الخليجية بالمنطقة ترتبط بمبررات التنافس على الموقع الاستراتيجي

والموارد وحماية المصالح الخليجية في المنطقة في ظل تحركات الاستقطاب الخليجية- الإيرانية

بالمنطقة.

Y -القرن الأفريقي في الحسابات الجيوسياسية التركية:

حيث تسعى تركيا للتواجد في العمق الأفريقي لتعزيز نشاطها الاقتصادي والسياسي، ولتوسيع نفوذها العسكري؛ حيث تقييم تركيا علاقات اقتصادية قوية مع الجانب الأثيوبي؛ إذ تعد رابع أكبر شريك تجارى لأنقرة في القارة السمراء، وتتلقى وحدها نحو ب, مليار دولار من إجمالي ج مليار دولار تستثرها تركيا في القارة، وفى مقابل هذا ينصب اهتمام تركيا الإنساني والعسكري كميج من الآداتين "الناعمة والصلبة" نحو الصومال؛ حيث بلغت المساعدات التركية الإنسانية والتقنية للصومال نحو

• . . مليار دولار بالإضـافة إلى حرصها على قيام قاعدة عسكرية تابعة لها في الصومال(10). ب-القرن الأفريقي في الحسابات الحيوسياسية الإسرائيلية: وكانت إسرائيل أبرز القوى الإقليمية المراهنة على حضورها بالمنطقة كإستراتيجية للأمن القومي الإسرائيلي من خلال تعزيز أمنها، وتحقيق الوصول لمنطقة شرق أفريقيا لتحسين العلاقات الإفريقية معها، وكسب أصواتها بالمنظمات الدولية، وكسر الحصار والعزلة المفروضة عليها إقليمياً، من خلال استثمار برامجها لتقديم المساعدات للدول النامية. وجاء استغلال إسرائيل لعلاقاتها المتميزة مع دول "كينيا وأوغندا وأثيوبيا" من أجل تأمين الطريق الملاحي الجوى إلى الثرق الأقصى وجنوب أفريقيا، وراهنت على هذه العلاقات السياسية والاقتصادية فقد استطاعت عقد مجموعة من الاتفاقيات العسكرية جاء أبرزها مع إريتريا بهدف مراقبة الملاحة بباب المندب ومراقبة الأنشطة الإيرانية(Tج). 
المحور الثالث: الهندسة الجيوسياسية والأفاق المستقبلية للقرن الأفريقي ما بعد أزمة

\section{كورونا.}

ألقت جائحة "كورونا COVID-19" الضوء على أهمية التفكير المستقبلي لنقلنا إلى ما وراء الافتراضات والمواقف التقليدية، والتكيف مع ما هو غير متوقع، ومن المؤكد أن هذه التداعيات كثفت

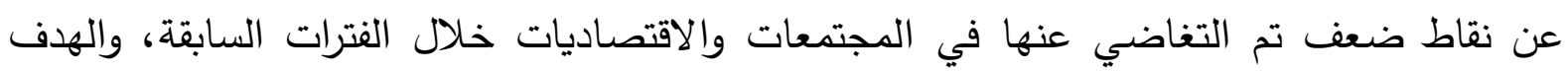
الأساسي من هذا المحور هو : إلقاء الضوء على مفهوم "الهندسة الجيوسياسية" كمدخل لإعادة الصياغة

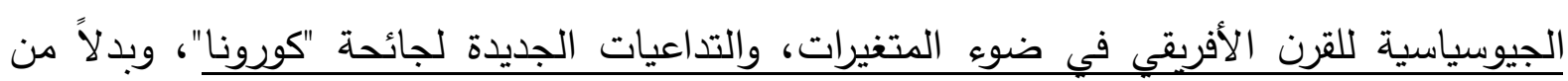
التوصل إلى استتتاجات "نهائية" أو وصف للتوصيات أو السياسات أو هيكل القوة، فإن هذا المحور يدعو للنظر في الكيفية التي يمكن من خلالها للمستقبل أن يتحدى الاحتمالات المقبولة للتأثير من القوى الفاعلة على الأمن الجيوسياسى لمنطقة القرن الأفريقي بعيدًا عن "نظام توازن القوى"؛ حيث يكون التغيير هو الأكثر احتمالًا، ويمكن القول بأنه في كل مستقبل بديل - بغض النظر عن مدى لتى

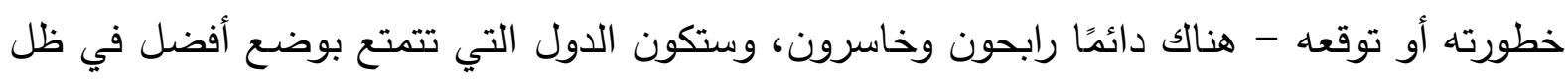
هذا النظام الجديد هي تلك الدول التي تتمتع بالقدرة على الإدراك والتعلم والتكيف حتى عندما تبدو

$$
\text { التوقعات القادمة غير مقبولة في الوقت الحاضر . }
$$

ومن ضمن المفاهيم الجديدة التي ظهرت في العلاقات الدولية في الآونة الأخيرة مفهوم "الهندسة الجيوسياسية"، والتي عرفت بأنها إعادة الهيكلة وخلق واقع سياسي واقتصادي وجيوسياسى جديد وفقاً لرؤبة جيوسياسية وجغرافية واقتصادية. أي أنه على دول القرن الأفريقي البحث عن بدائل أخرى بخلاف الاعتماد على التدخلات الخارجية لحل الأزمات التي تتعرض لها دول القرن الأفريقي. مسترشدة بما قامت به "الصين" في البحث عن بدائل جديدة رداً على محاولة الولايات المتحدة عزل "بكين" عبر اتفاقيات عبر المحيط الهادئ (TPP)، وعبر المحيط الأطلسي (TTIP)؛ فجاء الرد الصيني بـ "طريق الحرير الجديد"، والذي جاء معبراً عن رؤبة جيوسياسية وجغرافية واقتصادية واضحة من الصين في إطار البحث عن بدائل مستخدمة في ذلك كافة الإمكانيات والموارد المتاحة لديها(VI)، ولتحقيق ذلك ملك يجب تحليل السيناريوهات العالمية المحتملة في ظل جائحة "كورونا" لأخذها في الاعتبار عن صياغة الرؤية المستقبلية للقرن الأفربقي والتي تتمثل في: 
أولاً: الآفاق المستقيلية البديلة للمنافسة الجيوسياسية العالمية ما بعد أزمة كورونا.

لقد انتشر على الساحة الدولية ضرب من الجدل في كيفية التعاطى مع موضوع جائحة "كورونا" الفجائية، وكيفية الوقاية منها، وتباينت المواقف في هذا الثأن؛ فنجد أن هناك من هون من شأنها كالرئيس الأمريكي "ترامب" زاعماً أن من يروجون لهذا الخطر يقصدون من وراء ذلك ضرب الاقتصاد الأمريكي، أما رئيس الوزراء البريطاني "بوريس جونسون" فقد هون في البداية ثم تراجع أمام انتشار العدوى على نطاق أوسع، ويمكن القول بأن الجدل الذي يدور في هذا الثأن لا يتعلق بمجرد كونه خلاف سياسي أو علمي في كيفية التعاطي مع هذه الجائحة العالمية، بقدر ما يتعلق بالفلسفة العامة التي تقف خلف هذا النقاش السياسي الذي أغفل العديد من التوقعات المحتملة لهذا الوباء(^^). ومن هنا تأتى أهمية إعادة التفكير في الصياغة الجيوسياسية في ظل جائحة "كورونا" ويتم تناولها على هذا

ا-الرؤى والسيناريوهات المستقبلية للقوى الدولية الفاعلة في ضوء تحديات الوباء: في ضوء الدراسة التي قامت بها جامعة هاواى الأمريكية "مدرسة مانوا للدراسات المستقبلية" تم رصد أربعة رؤى استشرافية عالمية شاملة تمثلت في الآتي(ج): أ-الرؤيسة الاستثرافية العالمية الأولى: "التحول من الهيمنة إلى السيادة": حيث يرى هذا السيناريو أن "القوى العظمى" الجديدة هي تلك الدول التي حققت مستويات فائقة من المرونة الحيوية أمام منافسيها، من خلال البنية المعلوماتية بالإضافة إلى التميز في مجال الذكاء الاصطناعي، وتكون هي التي تستطيع أن تهيمن على المنافسة الجديدة. ب-الرؤية الاستشرافية العالمية الثانية: "الانهيار المنهجى" ويطلق عليها "فترة الدول المتحاربة الحديدة": حيث يصف العلماء العقد الثالث من القرن الحالي بأنه فترة "الدول المتحاربة الجديدة" - في إشارة إلى كل من حقبة الصراع الصيني القديم وكذلك القرن الحادي والعشرين القاري الأول، واندلاع الحرب الأهلية في الصين كثانى حرب أهلية في الصين في أقل من . . 1 عام- وأن العامل الأكثر إثارة الذى ساهم في عدم الاستقرار العالمي الجديد هو الأزمة التي أحدثها فيروس 
ج-الرؤية الاستشرافية العالمية الثالثة "الانضباط - الإقليمية الثمولية": حيث قد تؤدى الضغوط الثديدة على النظام الاجتماعي إلى دفع كل من الأنظمة الليبرالية والثمولية على حد سواء نحو

$$
\text { إجراءات شديدة المركزية والتقييد. }
$$

د-الرؤية الاستشرافية العالمية الرابعة "النمو المستمر": حيث تتمكن الدول التي لديها القدرة على

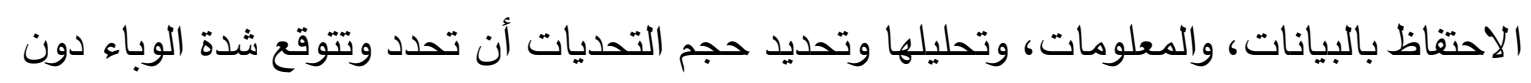
التنبؤ بطفرة تطوره.

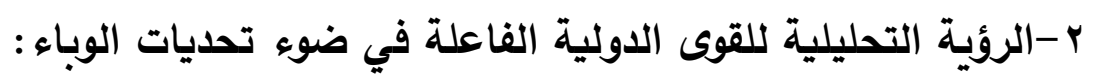
وفى إطار تحليل "الرؤى الأربعة الاستشرافية" نجد أنها صورة عامة للمستقبل تتكرر عبر التاريخ في صورها سالفة الذكر ، والتي تعد من أكثر المنهجيات المستخدمة على نطاق واسع لصياغة سيناريوهات الرؤى المستقبلية البديلة والتي يطلق عليها "العقود الأربعة الآجلة". ويوضح الثكل (1)

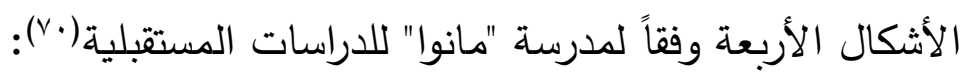

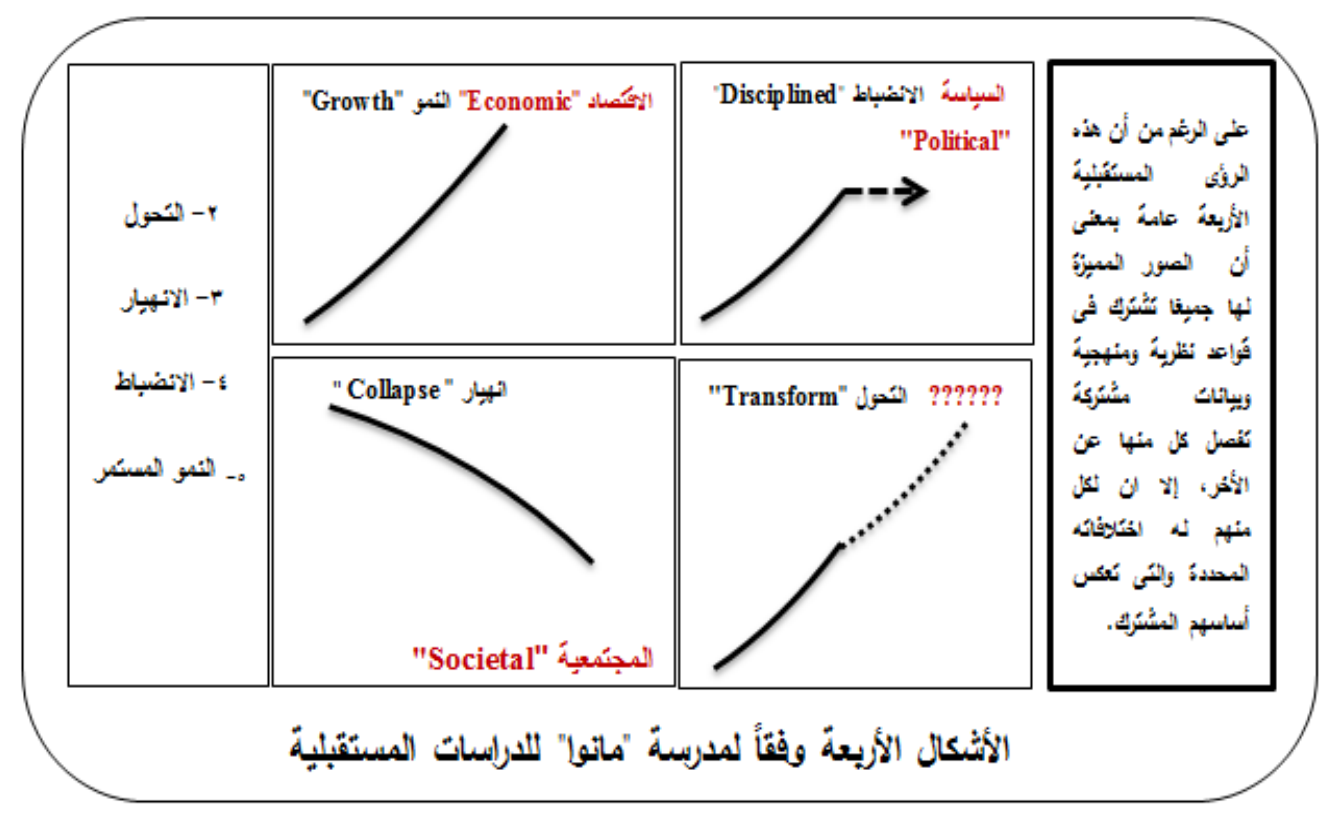

ويمكن القول بأن جميع الأثكال العامة الأربعة بالنسبة للقوى الفاعلة الدولية، لها احتمالات حدوث متساوية، وبالتالي يجب النظر إلى الجميع بنفس القدر من التركيز والاهتمام. والمتمعن في احتمالات حدوث أي من الأثكال الأربعة يجد أنها مرتبطة بعملية التخطيط، وقبل بضع سنوات، 
ابتكر "Wendy Schult" الرسم التخطيطى التالي لتوضيح انفتاح عملية المستقبل، وكذلك علاقته بالتخطيط بعيد المدى، والتخطيط اليومى - الإدارة اليومية ويوضح الشكل (Y) هذه العلاقة(V):

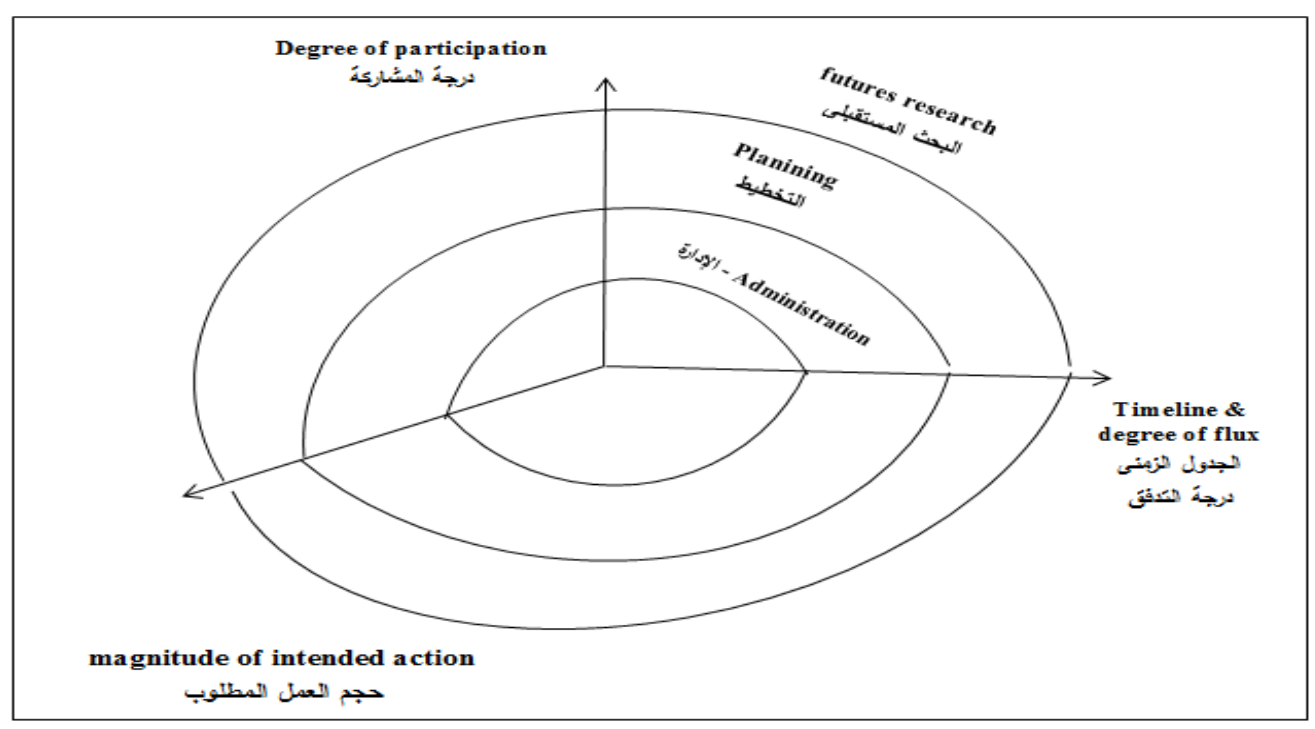

وأياً كانت الرؤية أو السيناريو المستقبلى للقوى الدولية الفاعلة والمتنافسة جيوسياسياً في عالم ما بعد "كورونا"، فإنه على دول منطقة القرن الأفريقي أخذ هذه السيناريوهات في الاعتبار عند صياغة الرؤية الجيوسياسية الجديدة للقرن الأفريقي بما يحقق الاستفادة منها. ثانيا: الآفاق المستقبلية البيلة للمنافسة الجيوسياسية في القرن الأفريقي ما بعد أزمة كورونا (الفرص والتحديات).

أظهرت أزمة" كورونا "الحاجة الماسة لإعادة دور الدولة للتدخل، والمساهمة لدعم الثركات ومساعدة الفئات المتضررة من الأزمة. أي أنه حينما تتعلق الأمور بمثل هذه الظروف تكتسب الدولة مشروعية التخخل سواء كان ذلك بالطريقة الناعمة أو بالطريقة الخشنه. 1-تحليل البيئة الداخلية لدول القرن الأفريقي:

أ-المميزات التي تتمتع بها منطقة القرن الأفريقي (نقاط القوة): يمكن رصد أهم نواحي القوة ذات الأبعاد السياسية والاقتصادية والعسكرية في النقاط التالية(Yr): - أنها تعد من أكثر المناطق الإقليمية أهمية بحكم موقعها، وثرواتها الطبيعية والجغرافية. - أنها تشرف على أكثر المسطحات المائية أهمية بالتكامل مع المنطقة العربية، سواء بالنسبة لاستراتيجيات القوى العالمية التي نشأت على مر التاريخ، أو بالنسبة لحركة التجارة العالمية. 
ب-التحديات التي تواجه منطقة القرن الأفريقي (نقاط الضعف): ويمكن رصد أبرز التحديات الداخلية ذات الأبعاد السياسية، والاقتصادية، والعسكرية، والاجتماعية المؤثرة على استقرار منطقة القرن الأفريقي في النقاط التالية(rr): (

- غياب الدور الحقيقي للدولة فلم تعد الدولة في منطقة القرن الأفريقي لها تأثر ودور واضح. - أن بعض الأنظمة السياسية تعانى من بعض الأزمات والتوترات الداخلية التي قد تؤدى إلى عدم الاستقرار ، ولعل أبرزها أزمة المشاركة السياسية. - الخلط بين مفهوم الأمن القومي والأمن الذاتي؛ حيث تعد الأزمة الأكثر تأثيراً على الاستقرار الأمني والسياسي. - محدودية الموارد المائية، والفقر المائي لبعض دول القرن الأفريقي؛ إذ ستسهم هذه الأزمة في تقليص فرص الاستقرار الأمني والسياسي في المنطقة، حتى في الدول التي تمر في أراضيها مصادر المياه، وستتعاظم هذه الأزمة في غياب التتسيق والتعاون والتكامل، وهى القيم اللازمة للتخطيط الاستراتيجي لاستغلال الفائض المائي الهائل الاستغلال الأمثل. - الزيادة في معدلات المواليد؛ حيث تثكل التحدي الأعظد لإحداث الاستقرار الأمني والسياسي فيها، في غياب إحداث التنمية الحقيقة على نحو عام، وفى مجالات التنمية البشرية إلى نحو خاص، إذا لم تشهد جميع مجتمعات دول القرن الأفريقي تحولاً ونمواً اقتصادياً، وثقافياً، وتكنولوجياً، يواكب ويترافق مع هذا النمو الديموجرافى. - عرضتها للتبعية السياسية والاقتصادية؛ حيث تفتقر جميع دول القرن الأفريقي إلى وجود قواعد صناعية ثقيلة، وغياب التقدم العلمي، والتقنى من ناحية أخرى. الأمر الذي أدى إلى تعاظم حاجتها إلى الدول الصناعية الكبرى لتلبية احتياجاتها من المعدات والأدوات، وبالتالي أصبح استقرارها السياسي والأمني عرضة للخطر ، إن لم تلبّ مطالب هذه القوى. - - افتقار معظم دول القرن إلى التكنولوجيا المتقدمة تحدياً آخراً يحول دون تقدم هذه الدول، مما أدى إلى الهجرات الواسعة غير الشرعية، وهجرات سكان الريف إلى الحضر مما أثر على الصناعات الصغيرة التي كانت قائمة على المنتجات الزراعية والحيوانية. r -تحليل البيئة الخارحية لاول القرن الأفريقي: أ-الفرص المتاحة أمام دول القرن الأفريقي: 
- القرن الأفريقي جيوبوليتكياً أكبر كثيراً من القرن الأفريقي جغرافياً؛ حيث يتغير وفقاً لاستراتيجيات ومصالح القوى العظمى والكبرى.

- أنها منطقة ترتبط ارتباطاً وثيقاً بالمنطقة العربية، خاصة دولها المتثاطئة على البحر الأحمر . - الاستفادة من تجارب التتمية المستدامة الناجحة لبعض الدول الأفريقية وعلى وجه الخصوص الدولة المصريـة.

\section{ب-التهيدات التي تواجه دول القرن الأفريقي:}

- التدخلات الخارجية بكافة صورها السياسية والاقتصادية والعسكرية، للعديد من القوى الفاعلة الدولية، والإقليمية تحت غطاء محاربة الإرهاب، وتأثير المحتوى الفكري للمنظور الجيوبوليتيكى لهذه القوى بصورة كبيرة على استقرار المنطقة، بما أثر على الأمن الجيوسياسى للمنطقة. - افتقار منطقة القرن الأفريقي، والمنطقة العربية، إلى سبل التتسيق، والتعاون، والتكامل، وهى القيم

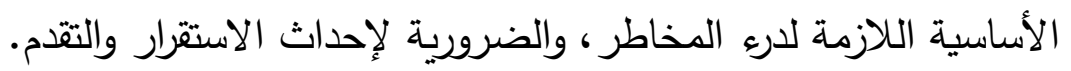
- عدم قدرة دول القرن الأفريقي عن تأسيس نظام إقليمي، بالصورة التي تجعل منها نظام إقليمياً مترابطاً بحكم التكامل والتماثل في عناصرها الجغرافية، والثقافية والاجتماعية واللغوية. - أنها غدت بؤرة تركيز، ومحط أنظار لاحتوائها على مصالح متعارضة متثابكه؛ بل ومسرح نثاط وتفاعلات هذه القوى وتلك الكيانات الاقتصادية العملاقة، فوجدت نفسها طرفاً في صراع القوى البحرية والقوى البرية في صورته الجديدة؛ حيث أصبحت في قلب ظاهرة التنافس الاستراتيجي. ب-التوجهات المستقبلية (المقترحة) لدول القرن الأفريقي: لما كانت الدراسة تسعى لإعادة الصياغة الجيوسياسية لمنطقة القرن الأفريقي، والاستفادة بمقدرات المنطقة الطبيعية، وفى ظل التطورات العالمية الحالية التي فرضتها جائحة "كورونا"، وبعد استعراض الرؤى والسيناريوهات المستقبلية للقوى الدولية الفاعلة في ضوء تحديات الوباء، ومن خلال دراسة وتحليل البيئة الداخلية والخارجية لدول المنطقة، يمكن القول بأنه إذا ما أرادت دول المنطقة إيجاد مكان في التنافسية العالمية الجديدة، يمكن الاستفادة من خلال تطبيق أحد الرؤى التالية أو الدمج بينها، والتي نوردها على النحو التالي: 
أ-اليؤية الاستشرافية الأولى:" التحول من التبعية إلى السيادة": ويتحقق ذلك من خلال تطيق الآليات التاليةة(؟)

- الآلية الأولم: ترتبط بالمنظور الأمني والسياسي: توسيع نطاق دول منطقة القرن الأفريقي لتشمل دولاً أخرى في القارة، وتفعيل دور الاتحاد الأفريقي في تنقية الأجواء في منطقة القرن الأفريقي، والعمل على إنهاء الخلافات، وفى نفس الوقت النظر في صياغة مبادرة تتضمن رؤيـة لنظام " إقليمي أمنى لادول منطقة القرن الأفريقي" تُراعَى فيه علاقات دول منطقة القرن الأفريقي يبعضها البعض، ومع دول الجوار ، على نحوِ يتم بموجبه إنهاء حالة العداء، والتوجه نحو ترسيخ العلاقات على أسس من علاقات حسن الجوار وعدم التدخل في الشئون الداخلية والأمن المشترك. - الآلية الثانية: ترتبط بالمنظور الاقتصادي: النظر في تكوين "هيئة إقليمية لاول القرن الأفريقي" تكون معنية بإدارة الموارد الطبيعية للدول، وإنشاء "منطقة إقليمية لوجستية"، والاستفادة من الخبرات المصرية في هذا الثأن، للاستفادة من الموقع اللوجستى والجيوبولوتيكى لدول المنطقة، وإقامة تجمع اقتصادي عربي أفريقي يضم دول المنطقة والدول العربية، للتحول نحو "الاقتصاد الأخضر" متخذاً من "الاقتصاد الأزرق" أساساً ومنطلقاً، في إطار برنامج الأمم المتحدة للتمية المستدامة . ب. rم، وبرنامج الاتحاد الأفريقي با • بام للتمية المستدامة؛ حيث تمتلك المنطقة ثروات هائلة بما لديها من بحار ومحيطات وبحيرات وأنهار • وتتعزز فرص إقامة مثل هذه المنطقة، بما تشهده المنطقة من تطورات إيجابية سبقت الإشارة إليها. ب-الرؤية الاستشرافية الثانية:"الانضباط - الإقليمية الشمولية": وتتمثل في الإجراءات شديدة المركزيـة والتقييد، أي: إعادة دور الدولة.

ج-الرؤيسة الاستشرافية الثالثة: "النمو المستمر": وهذه الرؤية لا تتحقق بمعزل عن الرؤية الأولى؛ حيث لا تستطيع دول المنطقة تحقيق ذلك إلا من خلال إحداث التنمية المستدامة التي تحققها الرؤية الأولى من خلال محورها الاقتصادي، والتي تتمكن الدول من خلالها إحداث التطور في المجال التكنولوجي، والصناعي بما يعطيها القدرة على الاحتفاظ بالبيانات والمعلومات وتحليلها وتحديد حجم التحديات أن تحدد وتتوقع الظواهر الغير طبيعية. 


\section{خاتمة الارزاسة :}

و يمكن تناول خاتمة الاراسة في النقاط التالية، للإجابة على تساؤل الاراسة الرئيسي وتساؤلاتها

الفرعية على النحو التالي: - التئ

أولاً: نتائج الدراستة:

حيث توصلت الدراسة إلى عدة نتائج من واقع الإشكالية الرئيسية والتي تم صياغتها لتحقيق أهداف الدراسة وهى : إلى أي مدى كان لايناميات القوى الفاعلة في منطقة القرن الأفريقي أثرها على الأمن الجيوسياسى للمنطقة؟، وكيف يمكن إعادة الصياغة الجيوسياسية لهذه المنطقة بما يحقق الأمن

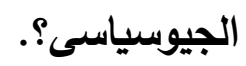

وخلاصة القول فإنه في ضوء ما تم استعراضه من واقع دراسة وتحليل "الأمن الجيوسياسى لمنطقة القرن الأفريقي وديناميات القوى الفاعلة، وصياغة الآفاق المستقبلية البديلة للمنافسة الجيوسياسية في القرن الأفربقي ما بعد جائحة "كورونا"، بدا واضحاً أهمية وخطورة أدوار "الفواعل الإقليمية والدولية" والتي أكدتها الأحداث خلال الدراسة، وتؤكدها كافة المؤشرات والمعطيات أن مجمل المتغيرات الراهنة في منطقة القرن الأفريقي في ظل وجود الفواعل الإقليمية والدولية تتجه نحو تحفيز الصراع واستمراره في المنطقة. ومن ثم فقد أضحت موازين القوة وما يرتبط بها من توازنات دولية وإقليمية، تمثل أحد العوامل المهمة لضرورة إعادة الصياغة الجيوسياسية للمنطقة، وتوسيع رقعة الخارطة الجيوبولتيكية أكثر لتشمل أغلب دول شرق أفريقيا وإقليم البحيرات العظمى، مؤكدة في ذلك على أن تطور الأحداث ومآلاتها المستقبلية في المنطقة، بات يعتمد بشكل أساسي على مدى تواجد الفواعل الإقليمية والدولية والتي أصبح دورها واضحاً في الفترة الأخيرة، من خلال استعراض نتائج الدراسة؛ حيث جاءت على النحو التالي : في استعراض المحور الأول: الديناميات الأمنية والجيوسياسية في القرن الأفريقي؛ تم التوصل إلى دنى النتائج التالية : - تمتع المنطقة بميزه تتافسية تؤكد الفرضية التي قامت عليها الدراسة، بأن الإقليم الذي تتواجد فيه الدولة يؤثر على حركتها السياسية، وهو ذاته نفس المفهوم الذي انطلقت منه أهداف النظريات

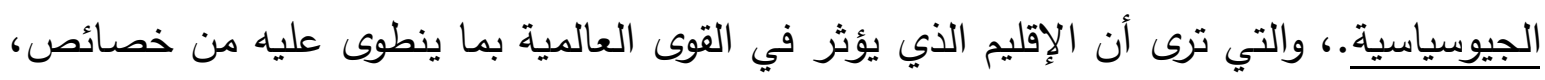
ومزايا تطلعًا نحو السيطرة العالمية، يكون موضع استقطاب، وجذب للعديد من هذه القوى، والتي 
ليست بالضرورة أن تكون متواجدة في هذا الإقليم، كما أن هذا التواجد قد أثر على الأمن الجيوسياسى للمنطقة، وهذا ما يؤكده المشهد العام في العقود الأخيرة. - يأتي اهتمام العديد من القوى الإقليمية والدولية، إلى اعتبارات جيوبولوتيكية وإستراتيجية، تتمثل في كونها معبر وشريان رئيسي للتجارة الدولية، وكونها ممراً للتحركات الأمنية لبعض القوى للمنطقة الكبرى المتجهة لمنطقة الشرق الأوسط والخليج العربي. وفى المحور الثاني : القرن الأفريقي في الحسابات الجيوسياسية الإقليمية والدولية، كانت النتائج كالثالي: - أن تحركات القوى الفاعلة تجاه القرن الأفريقي؛ والتي تحققت تحت غطاء مكافحة الإرهاب كانت بهدف السيطرة على منابع النفط في المنطقة، وما يؤكد ذلك ويرسخه ويعطى مؤشراً عليه، اشتداد الصراع بين الولايات المتحدة والصين على النفط في دول "القرن الأفريقي"، ومواجهة الولايات المتحدة، تصاعد النفوذ الروسي، والصيني في القارة وعلى وجه الخصوص الصراع على موارد الطاقة، والتنافس الحادث بين الولايات المتحدة وفرنسا في منطقة الشرق، والغرب الأفريقي حالياً، والمناطق التي تقع في إطار نطاق مسؤولية "قوة المهام المشتركة" لمنطقة القرن الأفريقي، وهى

$$
\text { (السودان، وأثيوبيا، وإريتريا، وجيبوتى، والصومال، وكينيا، وجزر سيشيل). }
$$
- أن التحركات الخليجية بالمنطقة ترتبط بمبررات التنافس على الموقع الاستراتيجي والموارد وحماية المصالح الخليجية في المنطقة في ظل تحركات الاستقطاب الخليجية- الإيرانية بالمنطقة. وفى المحور الثالث: الهندسة الجيوسياسية والأفاق المستقبلية للقرن الأفريقي ما بعد أزمة كورونا، كانت النتائج كالتالي :

- أن الجدل الذي يدور حول جائحة "كورونا" لا يتعلق بمجرد كونه خلاف سياسي أو علمي في كيفية التعاطي مع هذه الجائحة العالمية، بقدر ما يتعلق بالفلسفة العامة التي تقف خلف هذا النقاش الذي أغفل العديد من التوقعات المحتملة لهذا الوباء. - أن "القوى العظمى" الجديدة هي تلك الدول التي تستطيع أن تحقق مستويات فائقة من المرونة الحيوية أمام منافسيها، من خلال البنية المعلوماتية بالإضافة إلى التميز في مجال الذكاء الاصطناعي، وتكون هي التي تستطيع أن تهيمن على المنافسة الجديدة. 
- أن العامل الأكثر إثارة الذي ساهم في عدم الاستقرار العالمي الجديد هو الأزمة التي أحدثها فيروس

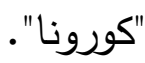

- قد تؤدى الضغوط الشديدة على النظام الاجتماعي إلى إجراءات شديدة المركزية والتقييد. - أن الدول التي لديها القدرة على الاحتفاظ بالبيانات، والمعلومات، وتحليلها وتحديد حجم التحديات أن تحدد وتتوقع شدة الوباء دون التنبؤ بطفرة تطوره.

ثانياً : توصيات الاراسة في ضوء النتائج والآفاق المستقبلية : وفى إطار ذلك فقد اقترحت الدراسة مجموعة من التوصيات تمثلت في: 1-من الناحية البنيوية:

- توسيع نطاق دول منطقة القرن الأفريقي لتشمل دولاً أخرى في القارة، وتفعيل دور الاتحاد الأفريقي في تنقية الأجواء في منطقة القرن الأفربقي، والعمل على إنهاء الخلافات.

- النظر في تكوين "هيئة إقليمية لدول القرن الأفريقي" تكون معنية بإدارة الموارد الطبيعية للدول، وإنشاء "منطقة إقليمية لوجستية"، والاستفادة من الخبرات المصرية في هذا الثأن، للاستفادة من الموقع اللوجستى والجيوبولوتيكى لدول المنطقة، وإقامة تجمع اقتصادي عربي أفريقي يضم دول المنطقة والدول العربية، ويتخذ من الاقتصاد الأزرق أساساً ومنطلقاً، في إطار برنامج الأمم المتحدة للتنمية المستدامة • ب. rم،

$$
\text { وبرنامج الاتحاد الأفريقي بr • ب للتنمية. }
$$

\section{Y-من الناحية القيمية:}

- التغلب على نزاعات الهوية من خلال تدعيم مفهوم الديمقراطية التوافقية، وإتاحة الفرصة للمشاركة

السياسية الحقيقية.

- التغلب على بؤر التوتر والانقسامات، من خلال النظر في صياغة مبادرة تتضمن رؤبة لنظام "إقليمي أمنى لدول منطقة القرن الأفريقي" تُراعَى فيه علاقات دول منطقة القرن الأفريقي يبعضها البعض، ومع دول الجوار على نحوِ يتم بموجبه إنهاء حالة العداء، والتوجه نحو ترسيخ العلاقات على أسس من علاقات حسن الجوار وعدم التدخل في الشئون الداخلية والأمن المشترك. - دفع كل من الأنظمة الليبرالية والشمولية على حد سواء نحو إجراءات شديدة المركزية والتقييد، أي اعادة دور الدولة. 


\section{مجلة كلية السياسة والاقتصساد العدد الحادي عشر - يوليو I.Y.}

- الدعوة إلى العمل الجاد نحو تغيير الأطر السياسية والاجتماعية والفكرية وتفعيل مفاهيم الحكم الرشيد والتتمية المستدامة من واقع الايكولوجيا السياسية الخاصة بكل نظام • الأهوامش و المراجح

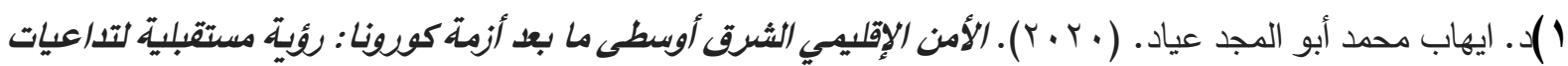
الحرب بالوكالةة. المؤتمر الدولي: منطقة الثرق الأوسط ما بعد أزمة كورونا: تحديات الوباء الجيواستراتيجى. (ط ا). برلين.

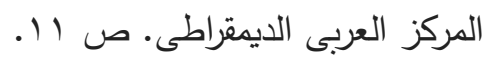

2)Kornely Kakachia,Stefan Meister, Benjamin Fricke, (eds).(2018). Geopolitics and Security: A New Strategy For The South Caucasus. Georgia. German Council on Foreign Relations.

3)A.F.K. Organski. (1958).World Politics. New York: Alfred A. Knopf.p277.

4)Madonna del Pantano, Lago Patria,(eds).(2019). Geopolitical Dynamics in the Horn of Africa and Mechanisms for Collaboration between Nato and Igad Countries. Addis Ababa: Institiute for Peace and Security Studies Addis Ababa University; Casier, T. 2016. 'From logic of competition to conflict: understanding the dynamics of EU-Russia relations'. Contemporary Politics, 22, 3, July; Sieg, H. M. 2016. Between Geopolitics and Transformation: Challenges and Perspectives for the Eastern Partnership. Berlin. DGAP Analysis 10. German Council on Foreign Relations; Magen, A. 2007. 'Transformative engagement through law: The acquis communautaire as an instrument of EU external influence', European Journal of Law Reform, 9, 3; Robert Chase. 1996. Emily Hill, Paul Kennedy, Pivotal States and U.S. Strategy. Foreign Affairs, volume 75 N 1; D. Helly, G. Galeazzi. 2015. Avant la lettre? The EU's comprehensive approach (to crisis) in the Sahel. ECDPM. Briefing note 75; Dias, A. M. 2008. An Inter-state War in 


\section{مجلة كلية السياسة والاقتصاد العدد الحادي عشر - يوليو ا.r.}

the Post-Cold War Era: Eritrea-Ethiopia (1998-2000), unpublished PhD thesis/Department of International Relations, London, London School of Economics and Political Science.

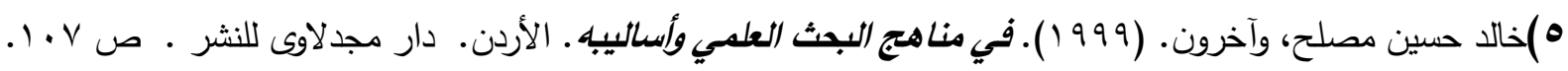
6)A.F.K. Organski. Ibid. p277; For more reading, see: Problems Of War and Peace. (1972). Moscow: Progress Publishers. P151.

7)Roy Macridis, Kenneth Thompson, (eds). (1982). Foreign Policy in World Politics. Prentice Hall. Inc., N.J. p2.

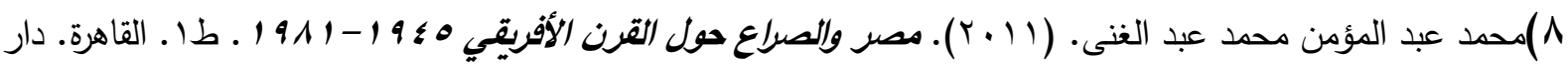

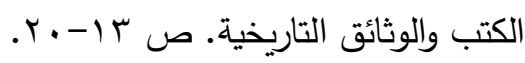

$$
\begin{aligned}
& \text { 9)عاصم فتح الرحمن. r ا ـ r. تغيير موازين القوى في القرن الأفريقي. الخرطوم. مركز دراسات المستقبل. ص } 10 \text { ا. }
\end{aligned}
$$

10)Alexander Randos. (2016). The Horn Africa Its Strategic Importance For Europe,

The Gulf States. And Beyond, Horizons, Center For International Relations And Sustainable Development, No 6. P: 150 .

$11)$ Ibid. P: 150-153.

12)Woodward, P. (1996). The Horn of Africa: state politics and international relations, London, I. B. Tauris.

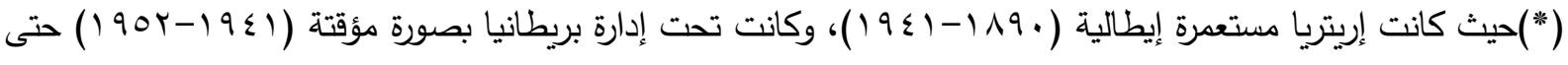

$$
\begin{aligned}
& \text { صدور القرار بمنح إريتريا الحكم الذاتي داخل اتحاد فيدرالي مع أثيوبيا (90 (19-197)؛؛ حيث صدر قرار بإلغاء الاتحاد } \\
& \text { عام با91 وتم دمج إريتريا كمقاطعة رقم (ع () للإمبراطورية الأثيوبية التي نتج عنها حرب دامت ثلاثة عقود من أجل } \\
& \text { الاستقلال في إريتريا ولم يتم التراجع عنه إلا في عام ا99 اـ راجع في ذلك: }
\end{aligned}
$$

-Dias, A. M. 2008:222; Kiflemariam Gebrewold, A. and S. Byrne. 2006:187. 
13)Dias, A. M. (2018). The Horn of Africa Pattern of Power: Continuity and Changes within the region and beyond (1998-2018).p 221-222. Abstract from 1st International Conference on Conflict Resolution and Peace Studies. UAL , Lisbon, Portugal. http://observare.ual.pt/crpsweb/en/abstracts-2018. For more reading, see: Kiflemariam Gebrewold, A. and S. Byrne (2006). "Small Arms and Light Weapons in the Horn of Africa", in D. A. Bekoe (ed.), East Africa and the Horn: Confronting Challenges to Good Governance, Colorado and London, Lynne Rienner Publishers, pp. 187-188.

14)Keller, E.J. (1997), “Rethinking African Regional Security”, in D.A. Lake and Patrick M. Morgan (eds ), Regional Orders: Building Security in a New World , Pennsylvania, Pennsylvania State University Press, pp. 296-317.

15)Woodward, P. Ibid. p20.

16)Lomo, Z. (2006). "Refugees in East Africa: Developing an Integrated Approach", in D. A. Bekoe (ed),(.East Africa and the Horn: Confronting Challenges to Good Governance ,Colorado and London, Lynne Rienner Publishers, pp. 37-57; For more reading, see: UNHCR. (2012). East and Horn of Africa Statistical Snapshot. available at :http://www.unhcr.org/pages/49e45a846.html ,accessed 19 January, 2021.

17)Clapham, C. (1996). "Boundary and Territory in the Horn of Africa", in P. Nuguent and A. I. Asiwaju (eds.), African Boundaries: Barriers, Conduits and Opportunities, London, Pinter, pp. 237-250.

18)Abbink, J. (2003). "Ethiopia- Eritrea: Proxy Wars and Prospects of Peace in the Horn of Africa”, Journal of Contemporary African Studies, 21(3), pp. 407-425.. 


\section{مجلة كلية السياسة والاقتصاد العدد الحادي عشر - يوليو ا.r.}

19)Cliffe, L. (1999). "Regional Dimensions of conflict in the Horn of Africa”, Third World Quaterly, 20(1), pp. 89-111; For more reading , see: Keller, E.J. Ibid. p297.

20)Markakis, J. (1998). Resource Conflict in the Horn of Africa, London, Thousand Oaks, New Delhi, PRIO and SAGE Publications; For more reading, see: Clapham, C. (2007).

"African Guerrillas Revisited", in M. Boas and Kevin C. Dunn (eds.), African Guerrillas: raging against the machine, Boulder and London, Lynne Rienner Publishers, pp. 221-233.

21) Clapham, C.( 2007). Ibid, pp. 221-233.

22)Kiflemariam Gebrewold, A. and S. Byrne. Ibid, p 186.

23)Cliffe, L.. Ibid, p 298.

24)Whitaker, Jennifer Seymour. (1981). Introduction:l'Afrique et les inte'rets arne'ricains, Les Etats - Unis et I'Afrique: les interets en Jeu, Paris.p.1-2.

25)Ibid.p.10-15.

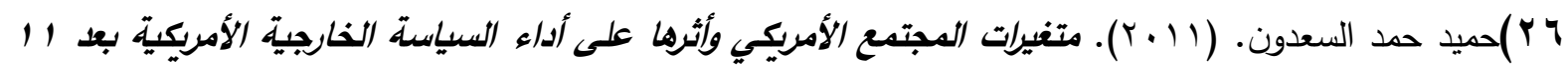
سبتمبر: الطبقة الوسطى انمونجا ـ بغداد. مجلة دراسات دولية. مركز الدراسات الدولية العدد(^ء) ، ص س؟ء. وللمزيد راجع

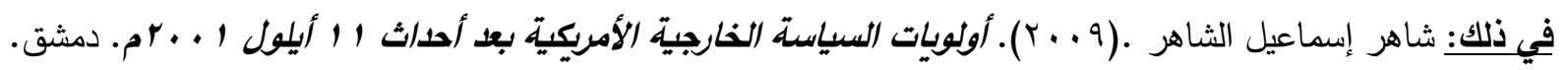
منشورات الهيئة العامة السورية للكتاب، وزارة الثقافة. ص Vـ

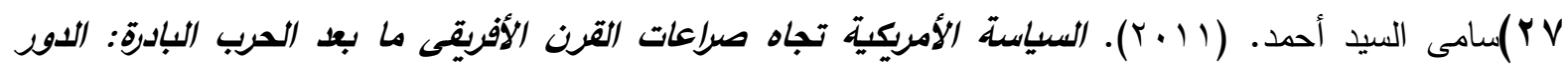
والاستجابة. طا ـ أبوظبى. مركز الإمارات للدراسات والبحوث الإستراتيجية. ص ع با -. ا. (*)(المصدر : الصراع الأمريكي الصيني في القرن الأفريقي. الجزء الثالث. مؤسسة الصومال الجديدة للإعلام والبحوث والتتمية.

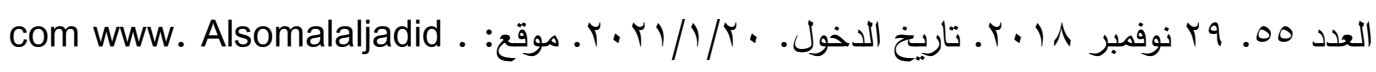


28)Richard.E.mashombs. (2000).Africa in the global economy. London: Lynne Rienner Publis hers, P.126-130. See too: Bp statistical Review of world Energy 2011, P.8-9-20-22. See Too: international energy agency report, oilmarket report, Op.Cit., P.19.

(")تُعد جزء من القيادة الأمريكية الأفريقية "افريكوم"، والتى تعمل من أجل تعزيز شراكات عسكرية إقليمية في منطقة شرق أفريقيا لتمكن الولايات المتحدة من فرض الهيمنة الأمنية في المنطقة على المدى الطويل، كما تقوم قوات المهام المشتركة من بناء صداقات وعلاقات في الدول الأفريقية، وخلق شراكات لتمكن أفريقيا من مواجهة تحدياتها الأمنية، وتحسين الأمن، وزيادة الاستقرار في منطقة "القرن الأفريقي" وشرق أفريقيا (عاصم فتح الرحمن، 11 ــ: 0).

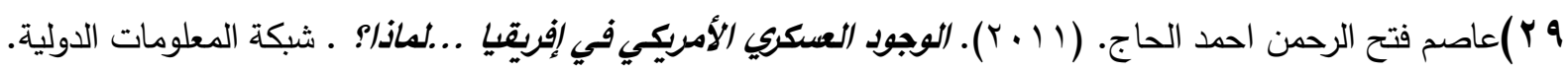

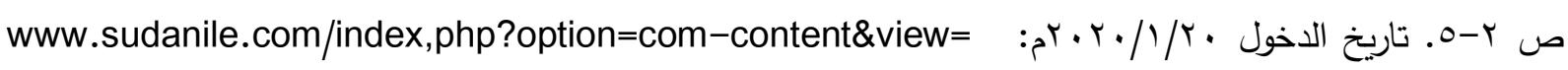
.article $\&=3853-02-26-10-20-05 \&$ catid $=977: 2011-5-24-08-47-1-\&$ Itemid $=55$ 30) office of the united states trade representative. (2021). AGOA Status in Africa. available at https://ustr.gov/countries-regions/africa/east-africa/eritrea.

$$
\text { (المصدر : تم إعداد البيانات من خلال: (الم: }
$$

office of the united states trade representative. (2021). AGOA Status in Africa. available at https://ustr.gov/countries-regions/africa/east-africa/eritrea.

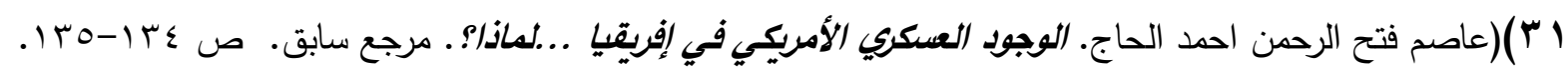

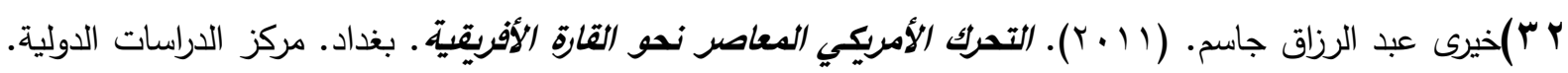
سلسلة دراسات إستراتيجية. العدد 711 ـ جامعة بغداد. ص • 9.

33)Whitaker, Jennifer Seymour. Ibid. p 15.

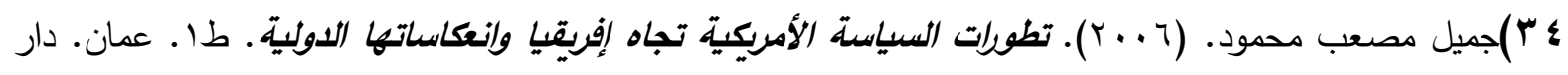
مجدلاوى. ص VT. 


\section{مجلة كلية السياسة والاقتصاد العدد الحادي عشر - يوليو Y.r.}

35)ultürk, Idemg Mehmet Cem. (2017). Russia's Renewed Interests in the Horn of Africa As a Traditional and Rising Power, Volume 2, Issue 1 Feb. pp. 121-143; For more reading, see: Kester Kenn Klomegah. (2016). why are Russians complaining about Africa?. Pambazuka News, Nov 17, available at https://goo.gl/GjLc8L.

36)Theodore Karasik and Giorgio Cafiero. (2017). Why Does Vladimir Putin Care About

Sudan?, Atlantic Council, NOVEMBER 27, available at https://goo.gl/jcKGPd.

37)Yacqub Ismail. (2018). Russia and Sudan are Cozying Up, 26 MAR, available at: https://goo.gl/XZcMUX.

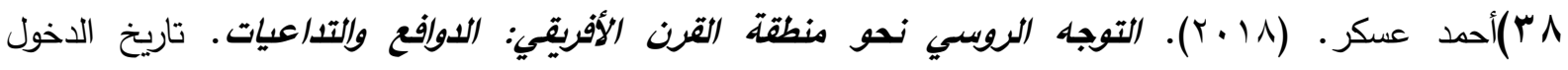
. https://www.qiraatafrican.com/home/new $1 / 23 . r \cdot r \cdot / / / r$.

39)Arkhangelskaya, A, \& Shubin, V 2013, 'Russia's Africa Policy', ohannesburg: South African Institute of International Affairs, SAllA Occasional Paper, No 157, September. P7.

40)Shubin, V (2010), 'Russia and Africa: Coming Back?', Russian Analytical Digest No.

83, 24 September, pp.4-7, retrieved 20 December. P5.

41 )Deich,T (2009), 'Politics as a Factor of Image of Russia in Africa', African Studies in Russia, Yearbook 2003-2007. Moscow. Russian Academy of Sciences Institute for African Studies, pp.135. retrieved 20 December

2016,http://www.inafran.ru/sites/default/files/page_file/ african _ studie _in_russia_yearbook_2003-2007.pdf.

Y \& أحمد عسكر . التوجه الروسي نحو منطقة القرن الأفريقي: الدوافع والتداعيات . مرجع سابق . 
43)Johan Burger. (2018). Russia in Africa: A return to old haunts?, 31 MAY, available at https://goo.gl/aepRmN.

44)Ibid.

$$
\text { ه ؛ أحمد عسكر . التوجه الروسى نحو منطقة القرن الأفريقي: الدوافع والتلداعيات. مرجع سابق. }
$$

46)Johan Burger. Russia in Africa: A return to old haunts?.Ibid.

47)Abdi Latif Dahir. (2018). Russia is the latest world power eyeing the Horn of Africa.

Quartz Africa. September 3. available at https://goo.gl/oCg66p.

48)) Gregory Alonso Pirio and Robert Pittelli. (2015). Putin and his Oligarchs in Africa:

The cramble for Economic and Military Leverage, Strategic Thinking on East Africa.

October 20. available at https://goo.gl/m9fNT2.

49)Ismail Akwel. (2018). Russia to pitch first camp in Africa with military base in Somaliland. April 19. available at https://goo.gl/ykV4DA.

$$
\begin{aligned}
& \text { • •) أحمد عسكر . التوجه الروسي نحو منطقة القرن الأفريقي: الدوافع والثتاعيات . مرجع سابق . }
\end{aligned}
$$

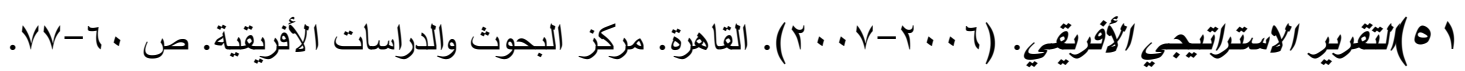

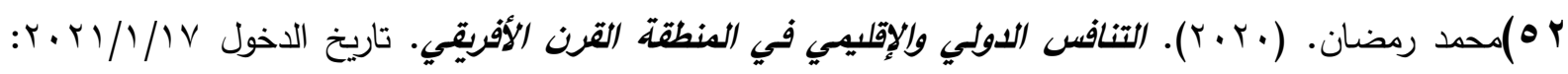

$$
\begin{aligned}
& \text {.http://arabprf.com/?p=2565 }
\end{aligned}
$$

54)Adama Gaye. 2008. La Chine en Afrique inquiète l'occident. New African. no. 3. pp. 8-9. 


\section{مجلة كلية السياسة والاقتصاد العدد الحادي عشر - يوليو I.Y.}

هـ)على حسين بكير • • ( • r. التنافس الجيو-استراتيجي للقوى الكبرى على موارد الطاقة: دبلوماسية الصين النفطية

$$
\text { الأبعاد والانعكاسات. بيروت. دار المنهل اللبناني للدراسات. ص ع (1) . ب |. }
$$

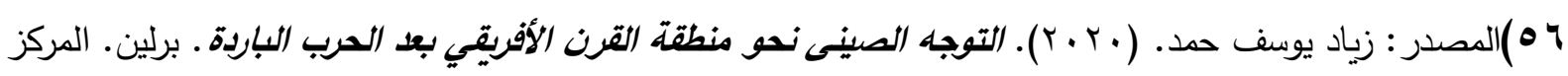
الديمقراطي العربي. المجلد ج. العدد V. يناير في: حورية توفيق مجاهد .9VY I. الدبلوماسية الصينية في القارة الأفريقية. القاهرة. مركز الأهرام للدراسات السياسية والإستراتجية. السياسة الدولية. العدد VV.

57)James tang. (2006). With the Grain or against the Grain : Energy Security and

Chinese Foreign Policy in the Hu Jintao. Washington, DC: The Brooking Institution, center for Northeast Asian policy Studies, October.

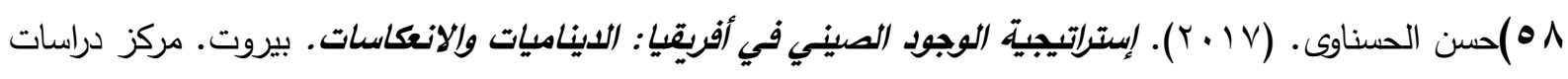
الوحدة العربية. مجلة المستقبل العربي العدد 77؟ ـ ديسمبر .

ه ه)أحمد، سامى السيد. (7 ( • ( ). التنافس الأمريكي الصيني في أفريقيا بعد الحرب الباردة. بغداد. المركز العراقى للدراسات

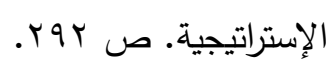

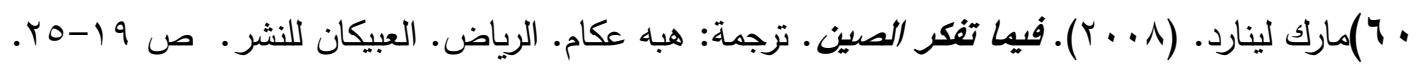

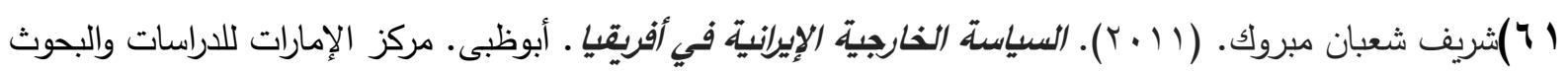

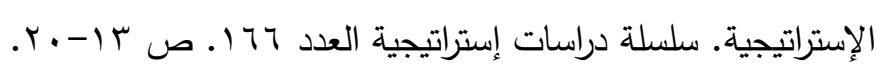

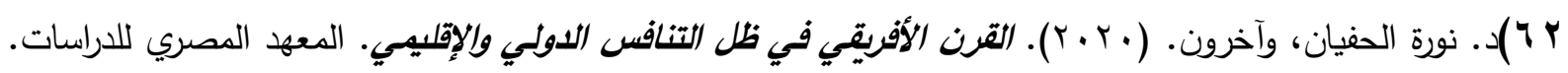

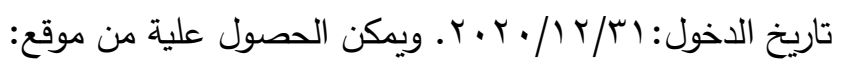
https://eipss-eg.org/\%D8\%A7\%D9\%84\%D9\%82\%D8\%B1\%D9\%86-\%D8.

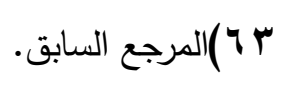

ع 7)محمد رمضان. التنافس الدولي والإقليسي في المنطقة القرن الأفربقي. مرجع سابق. 


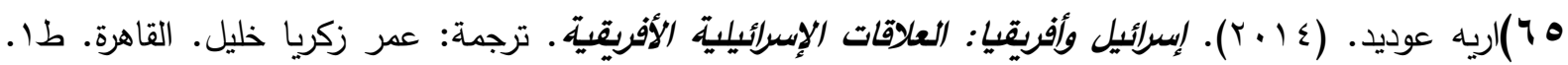
المؤسسة المصرية للتسويق والتوزيع. ص • r-O r.

66)Andrés Ortega November.( 2015). The New Silk Road: grand geopolitical engineering.

Global Spectator. Posted in Asia-Pacific, Global Policy, Global Spectator. 3/11/2015.https://blog.realinstitutoelcano.org/en/the-new-silk-road-grand-geopoliticalengineering/

67)Jacob S. Sotiriadis, Jairus Victor Grove, (2020). Global Future report: Alternative Futures of geoplitical Competition in A post-covid-19 world. Washington, D.C.,. Strategic Foresight and Futures Branch. June 2020. P 3-5. For more reading on futures studies, see :

-Wendell Bell ,Foundations of Futures Studies: Human Science for a New Era ,Volume 1, Transaction Publishers, 1997 ; Jim Dator ,World Futures Review ,Volume 7, Number 4, December 2015 ; Jennifer Gidley The Future: A very short introduction .Oxford University Press, 2017 ; Richard A. Lum, Steps to the Future: A Quick and Clean Guide to Creating Foresight,( Futurescribe: Honolulu, HI) 2016 ; Richard Slaughter ,Knowledge Base of Futures Studies, Revised 2020 ; Amy Webb , The Signals Are Talking: Why Today's Fringe is Tomorrow's Mainstream) ,PublicAffairs.

68)Ibid.

9 7)اريه عوديد. (ع ا • r). إسبائيل وأفريقيا: العلاقات الإسبائيلية الأفربقية. ترجمة: عمر زكريا خليل. القاهرة. طا. المؤسسة المصرية للتسويق والتوزيع. ص • r-O . 


\section{مجلة كلية السياسة والاقتصاد العدد الحادي عشر - يوليو ا.r.r.}

Jacob S. Sotiriadis, Jairus Victor Grove, (2020). Global Future report: Alternative (.

Futures of geoplitical Competition in $\boldsymbol{A}$ post-covid-19 world. Washington, D.C.,.

Strategic Foresight and Futures Branch. June 2020.p4.

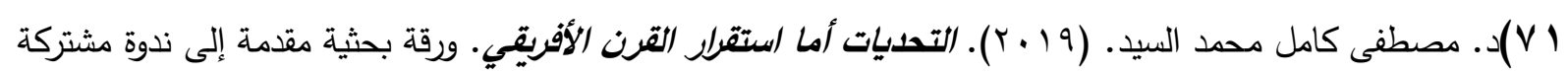
حول التطورات في منطقة القرن الأفريقي والبحر الأحمر . القاهرة. المجلس المصري للثؤون الخارجية. ص VY-Y

$$
\text { (V Y المصدر الثكل (Y) العلاقة بين المستقبل والتخطيط والإدارة: }
$$

Jim Dator. (2009), Alternative Futures at the Manoa School, Journal of Futures Studies, November, 14(2): $1-18$.

$$
\text { V V المرجع السابق. وللمزيد أيضاً حول الرؤية الاستشرافية يراجع في ذلك: }
$$

-Jacob S. Sotiriadis, Jairus Victor Grove, (2020). Global Future report: Alternative Futures of geoplitical Competition in A post-covid-19 world.

-Wendell Bell ,Foundations of Futures Studies: Human Science for a New Era ,Volume 1, Transaction Publishers, 1997.

-Jim Dator ,World Futures Review, Volume 7, Number 4, December 2015 ;Jennifer Gidley

The Future: $\boldsymbol{A}$ very short introduction .Oxford University Press,2017. 